\title{
Conjugacy Classes of the Hyperelliptic Mapping Class Group of Genus 2 and 3
}

\author{
Kazushi Ahara and Mitsuhiko Takasawa
}

\section{CONTENTS}

1. Introduction

2. Preliminaries

3. The Jones Representation

4. Theorems

5. Tables

Acknowledgements

Electronic Availability

References
Keywords: mapping class group, hyperelliptic, Siegel modular group, Jones representation, Meyer's function, Thurston type AMS Subject Classification: 57N05, 57M50
We present tables of conjugacy classes of the hyperelliptic mapping class group of genus 2 and 3, and some theorems on the Sp representation, the Jones representation, and Meyer's function.

\section{INTRODUCTION}

Let $\Sigma_{g}$ be a closed Riemann surface of genus $g$. Let $\mathcal{M}_{g}$ be the mapping class group of $\Sigma_{g}$, a finitely generated and finitely represented infinite group [Lickorish 1964; Humphries 1979; Wajnryb 1983; Matsumoto 2000]. Let $\sigma \in \mathcal{M}_{g}$ be the hyperelliptic involution (see Section 2 for definitions), and $\Delta_{g}$ the hyperelliptic mapping class group, that is, the centralizer of $\sigma$.

There are several important representations and 1-cocycles of $\Delta_{g}$. One is the fundamental linear representation or Sp-representation of $\mathcal{M}_{g}$, whose target is the Siegel modular group $\operatorname{Sp}(2 g, \mathbb{Z})$. Its kernel is the Torelli group $\mathcal{J}_{g}$. The second is Meyer's function $\varphi_{g}: \Delta_{g} \rightarrow \frac{1}{2 g+1} \mathbb{Z}$. This map is not a homomorphism, but the coboundary of $\varphi_{g}$ is called Meyer's signature cocycle $\tau_{g} \in Z^{2}\left(\mathcal{M}_{g}, \mathbb{Z}\right)$ [Meyer 1973]. The third is the Jones representation on $\Delta_{g}$, which arises from a representation of the Hecke algebra corresponding to a rectangular Young diagram [Jones 1987]. It is known how to get an explicit formula of the Jones representation [Kazhdan and Lusztig 1979; Wenzl 1988]. In the case $g=2$ Jones himself gave an explicit formula. We shall calculate an explicit representation in the case $g=3$ in the same way as Jones'. In fact, the Jones representation $\rho_{g}$ is given by maps $\rho_{g}: \Delta_{g} \rightarrow \widetilde{\mathrm{GL}}_{a}(\mathbb{Z}):=$ $\operatorname{GL}\left(a, \mathbb{Z}\left[q^{1 / a}, q^{-1 / a}\right]\right)$, where $a$ is an integer determined by $g$ and $\widetilde{\mathrm{GL}}_{a}(\mathbb{Z})$ is the set of $a \times a$ matrices with coefficients in the Laurent polynomial ring $\mathbb{Z}\left[q^{1 / a}, q^{-1 / a}\right]$. If $g=2,3,4$ we get $a=5,14,42$ 
respectively. Finally, any element of $\mathcal{M}_{g}$ is either periodic, reducible, or pseudo-Anosov. We call this classification the Thurston type [Thurston 1988].

This paper gives, for the cases $g=2,3$, a table of conjugacy classes of $\Delta_{g}$ up to word length 4 . The table contains the Sp-representation, Meyer's function, the Jones representation, and the Thurston type. We also calculate the table of some conjugacy classes of Torelli group $\mathcal{J}_{g}$.

This paper is organized as follows. Section 2 introduces notations on the Sp-representation and the Meyer's function. Section 3 discusses the Jones representation. Section 4 presents some theorems obtained from the tables. Section 5 lists the conjugacy classes.

\section{PRELIMINARIES}

Let $\mathcal{M}_{g}$ be the mapping class group of $\Sigma_{g}$, that is, the group of isotopy classes of orientation preserving diffeomorphisms of $\Sigma_{g}$. Let $C_{1}, C_{2}, \ldots, C_{2 g+1}, D$ be the simple closed curves on $\Sigma_{g}$ as follows:

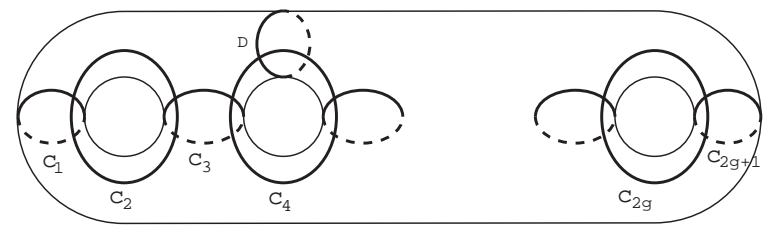

Let $\zeta_{1}, \zeta_{2}, \ldots, \zeta_{2 g+1}, \eta$ be the Dehn twists along $C_{1}, C_{2}, \ldots, C_{2 g+1}, D$; they generate $\mathcal{M}_{g}$ [Lickorish 1964; Humphries 1979]. The map

$$
\sigma:=\zeta_{1} \zeta_{2} \cdots \zeta_{2 g} \zeta_{2 g+1}^{2} \zeta_{2 g} \cdots \zeta_{2} \zeta_{1}
$$

satisfies $\sigma^{2}=1$ and is called the hyperelliptic involution. The centralizer

$$
\Delta_{g}:=\left\{\zeta \in \mathcal{M}_{g} \mid \zeta \sigma=\sigma \zeta\right\}
$$

of $\sigma$ is generated by $\zeta_{1}, \zeta_{2}, \ldots, \zeta_{2 g+1}$ : by [Birman and Hilden 1973], we have

$$
\begin{aligned}
& \Delta_{g}=\left\langle\zeta_{1}, \zeta_{2}, \ldots, \zeta_{2 g+1}\right| \zeta_{i} \zeta_{j}=\zeta_{j} \zeta_{i} \text { for }|i-j| \geq 2, \\
& \left.\quad \zeta_{i} \zeta_{i+1} \zeta_{i}=\zeta_{i+1} \zeta_{i} \zeta_{i+1}, \xi^{2 g+2}=1, \sigma^{2}=1, \sigma \zeta_{i}=\zeta_{i} \sigma\right\rangle,
\end{aligned}
$$

where $\xi=\zeta_{1} \zeta_{2} \cdots \zeta_{2 g+1}$. For $g=2, \eta=\zeta_{5}$ and hence $\Delta_{2}=\mathcal{M}_{2}$.

For any $\kappa \in \mathcal{M}_{g}$, define $C(\kappa): \mathcal{M}_{g} \rightarrow \mathcal{M}_{g}$ by

$$
C(\kappa)(\zeta)=\kappa \zeta \kappa^{-1} \text {. }
$$

Proposition 2.1. Let $\zeta_{a, b}:=\prod_{i=a}^{b} \zeta_{i}$ for $1 \leq a \leq b \leq$ $2 g+1$.
(1) For any $a, b$ such that $1 \leq a<b \leq 2 g+1$,

$$
C\left(\zeta_{a, b}\right)\left(\zeta_{i}\right)= \begin{cases}\zeta_{i} & \text { if } i<a-1, \\ \zeta_{i+1} & \text { if } a \leq i<b, \\ \zeta_{i} & \text { if } b+1<i .\end{cases}
$$

(2) $C\left(\zeta_{a, b}^{2}\right)\left(\zeta_{b}\right)=\zeta_{a}$.

(3) For any $a, b$ such that $1 \leq a<b \leq 2 g+1$, let $\eta_{a, b}:=\prod_{c=0}^{b-a} \zeta_{a, b-c}$. Then

$$
C\left(\eta_{a, b}\right)\left(\zeta_{i}\right)= \begin{cases}\zeta_{i} & \text { if } i<a-1, \\ \zeta_{a+b-i} & \text { if } a \leq i \leq b, \\ \zeta_{i} & \text { if } b+1<i\end{cases}
$$

(4) For any $\kappa, \zeta \in \mathcal{M}_{g}$, we have $C(\kappa) \circ C(\zeta)=C(\kappa \zeta)$, $C(\kappa)^{-1}=C\left(\kappa^{-1}\right)$, and $C(\kappa)\left(\zeta^{-1}\right)=(C(\kappa)(\zeta))^{-1}$.

(5) For any $t_{1}, t_{2}, \ldots, t_{2 g+1} \in \mathbb{Z}$ and $s \in S_{2 g+1}$ (the symmetric group of degree $2 g+1)$, we have

$$
\zeta_{1}^{t_{1}} \zeta_{2}^{t_{2}} \cdots \zeta_{2 g+1}^{t_{2 g+1}} \sim \zeta_{s(1)}^{t_{s(1)}} \zeta_{s(2)}^{t_{s(2)}} \cdots \zeta_{s(2 g+1)}^{t_{s(2 g+1)}}
$$

where $\sim$ means conjugacy in $\mathcal{M}_{g}$.

Proof. Parts (1), (2), (3) and (5) follow via straightforward computations from the relations of Birman and Hilden. Part (4) is trivial.

Because $\zeta \in \mathcal{M}_{g}$ is an isotopy class of homeomorphisms on $\Sigma_{g}, \zeta$ naturally induces a homomorphism

$$
\zeta_{*}: H_{1}\left(\Sigma_{g} ; \mathbb{Z}\right) \rightarrow H_{1}\left(\Sigma_{g} ; \mathbb{Z}\right) .
$$

Because rank $H_{1}=2 g$, we have $\zeta_{*} \in M_{2 g}(\mathbb{Z})$. It is known that $\zeta_{*}$ is contained in the Siegel modular group

$$
\operatorname{Sp}(2 g ; \mathbb{Z}):=\left\{X \in M_{2 g}(\mathbb{Z}) \mid{ }^{t} X J X=J\right\} .
$$

Here

$$
J=\left(\begin{array}{cc}
0 & E_{g} \\
-E_{g} & 0
\end{array}\right)
$$

for $E_{g}$ the $g \times g$ identity matrix. The map $\zeta \mapsto \zeta_{*}$ is a homomorphism

$$
\operatorname{Sp}: \mathcal{M}_{g} \rightarrow \operatorname{Sp}(2 g ; \mathbb{Z}): \zeta \mapsto \zeta_{*},
$$

called the Sp-representation.

For $\alpha, \beta \in \mathcal{M}_{g}$, let $A=\operatorname{Sp}(\alpha), B=\operatorname{Sp}(\beta)$. Define a real vector space

$$
\begin{aligned}
V_{A, B}=\left\{(x, y) \in \mathbb{R}^{2 g} \times \mathbb{R}^{2 g} \mid\right. & \left.\left(E_{2 g}-A^{-1}\right) x+\left(E_{2 g}-B\right) y=0\right\} .
\end{aligned}
$$

Let $\psi_{A, B}$ is a quadratic form on $V_{A, B}$ defined by

$$
\psi_{A, B}\left(\left(x_{1}, y_{1}\right),\left(x_{2}, y_{2}\right)\right):={ }^{t}\left(x_{1}+y_{1}\right) J\left(E_{2 g}-B\right) y_{2}
$$


Then $\psi_{A, B}$ is a symmetric form; we define the signature cocycle $\tau_{g}$ by

$$
\tau_{g}(\alpha, \beta):=\operatorname{sgn} \psi_{A, B}
$$

Lemma 2.2. (1) $\tau_{g} \in Z^{2}\left(\mathcal{M}_{g} ; \mathbb{Z}\right)$. That is, for all $\alpha, \beta, \gamma \in \mathcal{M}_{g}$, we have

$$
\tau_{g}(\beta, \gamma)-\tau_{g}(\alpha \beta, \gamma)+\tau_{g}(\alpha, \beta \gamma)-\tau_{g}(\alpha, \beta)=0 .
$$

(2) $\tau_{g}(\alpha, 1)=\tau_{g}\left(\alpha, \alpha^{-1}\right)=0$.

(3) $\tau_{g}(\alpha, \beta)=\tau_{g}(\beta, \alpha)$.

(4) $\tau_{g}\left(\alpha^{-1}, \beta^{-1}\right)=-\tau_{g}(\alpha, \beta)$.

(5) $\tau_{g}\left(\gamma \alpha \gamma^{-1}, \gamma \beta \gamma^{-1}\right)=\tau_{g}(\alpha, \beta)$.

This follows easily from the definition of $\tau_{g}$.

We have $(2 g+1) \tau_{g} \in B^{2}\left(\Delta_{g} ; \mathbb{Z}\right)$ [Endo 2000]. Therefore there is a function $\varphi_{g}: \Delta_{g} \rightarrow \frac{1}{2 g+1} \mathbb{Z}$ satisfying

$$
\delta \varphi_{g}=\tau_{g} \mid \Delta_{g}
$$

That is, for any $A, B \in \Delta_{g}$,

$$
\varphi_{g}(B)-\varphi_{g}(A B)+\varphi_{g}(A)=\tau_{g}(A, B)
$$

This $\varphi_{g}$ is called Meyer's function of genus $g$.

Lemma 2.3. Let $\alpha, \beta \in \Delta_{g}$.

(1) $\varphi_{g}(1)=0$.

(2) $\varphi_{g}\left(\alpha^{-1}\right)=-\varphi_{g}(\alpha)$.

(3) $\varphi_{g}\left(\beta \alpha \beta^{-1}\right)=\varphi_{g}(\alpha)$

The proof is straightforward from Lemma 2.2.

The next lemma gives the explicit value of Meyer's function.

Lemma 2.4. (1) $\varphi_{g}\left(\zeta_{i}\right)=(g+1) /(2 g+1)$.

$$
\varphi_{g}\left(\zeta_{i_{1}} \cdots \zeta_{i_{r}}\right)=\frac{r(g+1)}{2 g+1}-\sum_{j=1}^{r-1} \tau_{g}\left(\zeta_{i_{1}} \cdots \zeta_{i_{j}}, \zeta_{i_{j+1}}\right)
$$

For the proof of (1), see [Endo 2000]. Part (2) follows easily from (1).

\section{THE JONES REPRESENTATION}

Let $H(q, n)$ be the Hecke algebra of type $A_{n-1}$. That is, $H(q, n)$ is an algebra over $\mathbb{Z}\left[q, q^{-1}\right]$ generated by $g_{1}, g_{2}, \ldots, g_{n-1}$ with relations $\left(g_{i}-q\right)\left(g_{i}+1\right)=0$ for $i=1, \ldots, n-1, \quad g_{i} g_{i+1} g_{i}=g_{i+1} g_{i} g_{i+1}$ for $i=$ $1, \ldots, n-2$, and $g_{i} g_{j}=g_{j} g_{i}$ for $|i-j| \geq 2$. Jones [1987] showed that, if we regard $q$ as a complex number close to 1 , the irreducible representations of $H(q, n)$ are in one-to-one correspondence with
Young diagrams. He also showed that if we adjust the representation so that $\rho\left(g_{1} g_{2} \cdots g_{n-1}\right)^{n}=$ 1 , then $\rho$ is a representation of $\Delta_{g}$ if and only if $n=2 g+2$ and the Young diagram is rectangular. Therefore such a representation of $\Delta_{g}$ is called Jones representation and is denoted by $\rho_{g}$. Jones representations are in one-to-one correspondence with rectangular Young diagrams of size $2 g+2$.

In this section we shall obtain Jones representation explicitly using the Kazhdan-Lusztig formalism of W-graphs in case $g=2,3$. Kazhdan and Lusztig [1979] introduced W-graphs that allow us to get a representation of $H(q, n)$. But, as Jones points out, a simple way to go from a Young diagram to a Wgraph seems to be lacking in [Kazhdan and Lusztig 1979]. Ochiai and Kako [1995] wrote software to list up all of irreducible representations of $H(q, n)$. Using this software one can get the complete correspondence between Young diagrams and W-graphs, and in particular the $\mathrm{W}$-graphs corresponding to $2 \times 3$ and $2 \times 4$ rectangular Young diagrams:
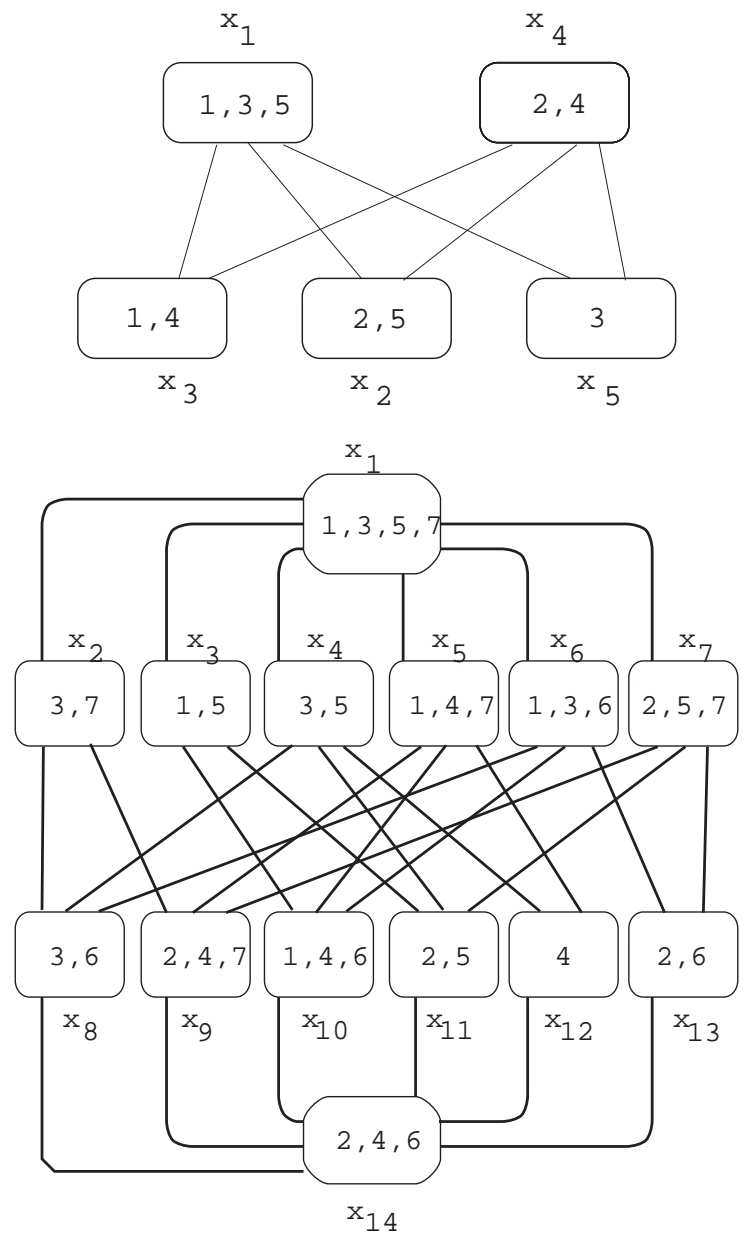
Let $\left\{x_{1}, \ldots, x_{a}\right\}$ be vertices of the W-graphs. We assign a subset of $\{1,2, \ldots, 2 g+1\}$ to each vertex. Let $X_{i}$ be the set assigned to $x_{i}$.

If two vertices $x_{j}$ and $x_{k}$ are connected by an edge, we assign two Laurent polynomials $\mu(j, k)$ and $\mu(k, j)$ to the edge.

Let $V$ be a vector space over $\mathbb{Z}\left[q, q^{-1}\right]$ spanned by $\left\{x_{1}, \ldots, x_{a}\right\}$, and define nondegenerate matrices $\rho_{g}\left(\zeta_{i}\right): V \rightarrow V$ by

$$
\rho_{g}\left(\zeta_{i}\right)\left(x_{j}\right)= \begin{cases}q x_{j} & \text { if } i \in X_{j}, \\ -x_{j}+\sum_{k:(*)} \mu(j, k) x_{k} & \text { if } i \notin X_{j},\end{cases}
$$

where $k:(*)$ means that there is an edge $\overline{x_{j} x_{k}}$ and that $i \in X_{k}$.

Kazhdan and Lusztig [1979] give the values of $\mu(j, k)$, but we shall recompute them. This is not hard to do if we use the relations among $\zeta_{i}$ 's. First we obtain

$$
\mu(j, k) \mu(k, j)=q .
$$

To illustrate this, take $g=2, j=2, k=5$. Then

$$
\begin{aligned}
\rho_{2}\left(\zeta_{1}\right) \rho_{2}\left(\zeta_{2}\right) \rho_{2}\left(\zeta_{1}\right)\left(x_{5}\right) & =(1-\mu(2,5) \mu(5,2)) x_{5} \\
+\mu(5,2)(-2 q+\mu(5,2) \mu(2,5)) x_{2}, & \\
\rho_{2}\left(\zeta_{2}\right) \rho_{2}\left(\zeta_{1}\right) \rho_{2}\left(\zeta_{2}\right)\left(x_{5}\right)= & \left(-q^{2}+q \mu(5,2) \mu(2,5)\right) x_{5}
\end{aligned}
$$$$
-q \mu(5,2) x_{2} \text {. }
$$

Thus $\mu(2,5) \mu(5,2)=q$.

Next, if $\left\{x_{i}, x_{j}, x_{k}, x_{l}\right\}$ is a simple closed path of length 4 on the $\mathrm{W}$-graph, then

$$
\mu(i, j) \mu(j, k)=\mu(i, l) \mu(l, k) .
$$

Again as an illustration, take $g=2$ and $(i, j, k, l)=$ $(1,2,5,4)$. Then

$$
\begin{aligned}
\rho_{2}\left(\zeta_{2}\right) \rho_{2}\left(\zeta_{4}\right)\left(x_{1}\right)=x_{1}-\mu(1,4) x_{4} & -\mu(1,2) x_{2} \\
& +\mu(1,2) \mu(2,5) x_{5}, \\
\rho_{2}\left(\zeta_{4}\right) \rho_{2}\left(\zeta_{2}\right)\left(x_{1}\right)=x_{1}-\mu(1,4) x_{4} & -\mu(1,2) x_{2} \\
& +\mu(1,4) \mu(4,5) x_{5} .
\end{aligned}
$$

Hence $\mu(1,2) \mu(2,5)=\mu(1,4) \mu(4,5)$.

It is easy to check that for any $\mu(j, k)$ we have

$$
\left(\rho_{g}\left(\zeta_{i}\right)-q E_{a}\right)\left(\rho_{g}\left(\zeta_{i}\right)+E_{a}\right)=0
$$

for any $i$. We cannot determine $\mu(j, k)$ uniquely. But any choice must allow us to get the same representation. For example, it is easy to understand the following assignment. In the case $g=2$, let $A=\left\{x_{1}, x_{3}\right\}$, and $B=\left\{x_{2}, x_{4}, x_{5}\right\}$. We observe that the $\mathrm{W}$-graph is a (complete) dipartite graph.
Using this feature, define $\mu(j, k)$ by

$$
\mu(j, k)= \begin{cases}q & \text { if } x_{j} \in A, x_{k} \in B, \\ 1 & \text { if } x_{j} \in B, x_{k} \in A .\end{cases}
$$

Jones [1987] obtains matrices of $\rho_{2}\left(\zeta_{i}\right)$ in this way. In the same fashion, in the case $g=3$, let

$$
\begin{aligned}
& A=\left\{x_{1}, x_{8}, x_{9}, x_{10}, x_{11}, x_{12}, x_{13}\right\}, \\
& B=\left\{x_{2}, x_{3}, x_{4}, x_{5}, x_{6}, x_{7}, x_{14}\right\} .
\end{aligned}
$$

Define $\mu(j, k)$ as above. See formulas for $\rho_{3}$ in Section $5 \mathrm{C}$.

\section{THEOREMS}

Theorem 4.1. For any $\zeta \in \mathcal{M}_{g}$,

$$
\operatorname{det}\left(y E_{2 g}-\operatorname{Sp}(\zeta)\right)=\operatorname{det}\left(y E_{2 g}-\operatorname{Sp}\left(\zeta^{-1}\right)\right) .
$$

Proof. For $\zeta \in \mathcal{M}_{g}$, we have $J \operatorname{Sp}(\zeta) J^{-1}={ }^{t} \operatorname{Sp}\left(\zeta^{-1}\right)$. Therefore

$$
\begin{aligned}
\operatorname{det}\left(y E_{2 g}-\operatorname{Sp}(\zeta)\right) & =\operatorname{det}\left(y E_{2 g}-{ }^{t} \operatorname{Sp}\left(\zeta^{-1}\right)\right) \\
& =\operatorname{det}\left(y E_{2 g}-\operatorname{Sp}\left(\zeta^{-1}\right)\right) .
\end{aligned}
$$

The following corollary is equivalent to this theorem.

Corollary 4.2. For $\zeta \in \mathcal{M}_{g}$, suppose that the characteristic function of $\mathrm{Sp}(\zeta)$ is given by

$$
\operatorname{det}\left(y E_{2 g}-\operatorname{Sp}(\zeta)\right)=\sum_{i=0}^{2 g} s_{i} y^{i} .
$$

Then $s_{i}=s_{2 g-i}$.

We now show that the characteristic function of the Jones representation also has a symmetry as that of the Sp-representation.

Theorem 4.3. Let $g=2,3$ or 4 . For any $\zeta \in \Delta_{g}$, if $J(\zeta)$ is given by $J(\zeta)(y, q):=\operatorname{det}\left(y E_{a}-\rho_{g}(\zeta)\right)$, then

$$
J(\zeta)(y, q)=J\left(\zeta^{-1}\right)\left(y, q^{-1}\right),
$$

where $a=a(g)$ is the size of the Jones representation of genus $g$.

Proof. Define $\bar{\rho}_{g}: \Delta_{g} \rightarrow \widetilde{\mathrm{GL}}_{a}(\mathbb{Z})$ by

$$
\bar{\rho}_{g}(\zeta):={ }^{t}\left(\overline{\rho_{g}(\zeta)}\right)^{-1} \text {. }
$$

Here $X \mapsto \bar{X}: \widetilde{\mathrm{GL}}_{a}(\mathbb{Z}) \rightarrow \widetilde{\mathrm{GL}}_{a}(\mathbb{Z})$ is an automorphism induced from a map $q \mapsto q^{-1}$. Clearly $\bar{\rho}_{g}$ is an irreducible representation of $\Delta_{g}$. In fact, $\bar{\rho}_{g}$ is a representation because

$$
\begin{aligned}
\bar{\rho}_{g}(\zeta \eta) & ={ }^{t}\left(\overline{\rho_{g}(\zeta \eta)}\right)^{-1} \\
& ={ }^{t}\left(\overline{\rho_{g}(\zeta)}\right)^{-1 t}\left(\overline{\rho_{g}(\eta)}\right)^{-1}=\bar{\rho}_{g}(\zeta) \bar{\rho}_{g}(\eta) .
\end{aligned}
$$


Irreducibility can be shown easily. Jones [1987] has shown that the simple $H(q, n)$ modules are in oneto-one correspondence with Young diagrams (if $q$ is close to 1), and that it defines a representation of $\Delta_{g}$ if and only if the Young diagram is rectangular. It follows that $\rho_{g}$ and $\bar{\rho}_{g}$ are the same representation. That is, there is a nonsingular matrix $P \in \widetilde{\mathrm{GL}}_{a}(\mathbb{Z})$ such that $P$ has an inverse matrix $P^{-1}$ with $\operatorname{det}(P) P^{-1} \in \widetilde{\mathrm{GL}}_{a}(\mathbb{Z})$, and $P \rho_{g}(\zeta) P^{-1}=\bar{\rho}_{g}(\zeta)$ for any $\zeta$. Therefore

$$
\begin{aligned}
J(\zeta)(y, q) & =\operatorname{det}\left(y E_{a}-\rho_{g}(\zeta)\right)=\operatorname{det}\left(y E_{a}-\bar{\rho}(\zeta)\right) \\
& =\operatorname{det}\left(y E_{a}-{\overline{\rho_{g}}(\zeta)}^{-1}\right)=J\left(\zeta^{-1}\right)\left(y, q^{-1}\right) .
\end{aligned}
$$

In the case $g=2$, we can get $P$ explicitly. It is easily shown that such $P$ is determined uniquely up to constant multiplication and equals

$$
\left(\begin{array}{ccccc}
(q+1)^{2} & -q(q+1) & 2 q & -q(q+1) & -q(q+1) \\
-q-1 & q^{2}+q+1 & -q-1 & q & q \\
2 q & -q(q+1) & (q+1)^{2} & -q(q+1) & -q(q+1) \\
-q-1 & q & -q-1 & q^{2}+q+1 & q \\
-q-1 & q & -q-1 & q & q^{2}+q+1
\end{array}\right) .
$$

Remark. The target space of the Sp-representation is the Siegel modular group $\operatorname{Sp}(2 g, \mathbb{Z})$. This allows us to show that the target of the Jones representation is contained in an extension of the Siegel modular group. That is, let

$$
\operatorname{Sp}(q, g):=\left\{A \in{\widetilde{\mathrm{GL}_{a}}}_{a}(\mathbb{Z}) \mid{ }^{t} \bar{A} P A=P\right\} .
$$

It is easy to show that Theorem 4.3 and the following corollary are equivalent. Remark that $\operatorname{det}\left(\rho_{g}\left(\zeta_{i}\right)\right)=$ -1 for $g=2,3$. See the formulas of $\rho_{g}$ in [Jones 1987] and Section 5C.

Corollary 4.4. Let $g=2,3$. For $\zeta \in \Delta_{g}$, suppose that the characteristic function of $\rho_{g}(\zeta)$ is given by

$$
\operatorname{det}\left(y E_{a}-\rho_{g}(\zeta)\right)=\sum_{i=0}^{a} J_{i}(q) y^{i} .
$$

Here $a=a(g)$ is the size of the Jones representation and $J_{i}(q)$ is a Laurent polynomial of $q^{1 / a}$. Then

$$
J_{i}(q)=(-1)^{a \varepsilon(\zeta)} J_{a-i}\left(q^{-1}\right),
$$

where $\varepsilon(\zeta)$ is the parity of the word length of $\zeta$.

There is a relationship between the Jones representation and Meyer's function:

Theorem 4.5. If $g=2$,

$$
q^{-\varphi_{g}(\zeta)} \rho_{g}(\zeta) \in \operatorname{GL}\left(a, \mathbb{Z}\left[q, q^{-1}\right]\right) .
$$

Proof. For any $A, B \in \Delta_{g}$, we have $\varphi_{g}(B)-\varphi_{g}(A B)+$ $\varphi_{g}(A)=\tau_{g}(A, B)$ by definition. Therefore $\varphi_{g} \bmod$. $\mathbb{Z}$ is a homomorphism. It follows that it is sufficient to show Theorem 4.5 for generators $\zeta_{i}$. We do it by a straightforward calculation in the case $g=2$.

Remark. For higher genus we don't have a similar theorem. This theorem implies that the abelianization of $\Delta_{2}$ contains $\mathbb{Z} / 5 \mathbb{Z}$. (If fact, $\Delta_{2}^{\text {abel }}=\mathbb{Z} / 10 \mathbb{Z}$.) Therefore Theorem 4.5 asserts nothing new. But it remains an open problem whether or not Meyer's function is wholly contained in the Jones representation.

We now consider a modified characteristic function of the Jones representation. Suppose $g=2$ and set

$$
\tilde{J}(\zeta)(y, q):=\operatorname{det}\left(y q^{\varphi_{g}(\zeta)} E_{a}-\rho_{g}(\zeta)\right) \in \mathbb{Z}\left[y, q, q^{-1}\right] .
$$

Corollary 4.6. $\tilde{J}(\zeta)(y, q)=\tilde{J}\left(\zeta^{-1}\right)\left(y, q^{-1}\right)$.

This follows from Lemma 4.4 and Lemma 2.3(2).

Finally, let inv : $\Delta_{g} \rightarrow \Delta_{g}$ be a map defined by

$$
\operatorname{inv}\left(\zeta_{i_{1}} \zeta_{i_{2}} \cdots \zeta_{i_{n}}\right):=\zeta_{i_{n}} \cdots \zeta_{i_{2}} \zeta_{i_{1}} .
$$

This map is well defined because Birman-Hilden relations of $\Delta_{g}$ are invariant under inv. (Note that $\xi=$ $\zeta_{1} \cdots \zeta_{2 g+1}$ is conjugate to $\xi^{\prime}=\operatorname{inv}(\xi)$ via $C\left(\eta_{1,2 g+1}\right)$. See Proposition 2.1(3).)

Theorem 4.7. If $g=2,3,4$, then

$$
J(\zeta)(y, q)=J(\operatorname{inv}(\zeta))(y, q)
$$

for any $\zeta \in \Delta_{g}$.

Proof. Define $\hat{\rho}_{g}: \Delta_{g} \rightarrow \widetilde{M}_{a}(\mathbb{Z})$ by

$$
\hat{\rho}_{g}(\zeta):={ }^{t}\left(\rho_{g}(\operatorname{inv}(\zeta))\right)
$$

It is easy to show that $\hat{\rho}_{g}$ is also a representation of $\Delta_{g}$ and $H(q, 2 g+1)$, and it is irreducible. We can conclude that $J(\zeta)=J(\operatorname{inv}(\zeta))$ in the same way as the proof of Theorem 4.3.

Remark. It is an open problem whether the kernel of the Jones representation is generated by only the hyperelliptic involution $\sigma$. Using the preceding theorem, we cannot answer this question straightforward but we can have an evidence such that there are at least two conjugacy classes of $\Delta_{g}$ such that 
they cannot distinguish by $J(\zeta)$. For example, $g=2$ and let

$$
\begin{aligned}
\zeta_{6} & =\left(\zeta_{1} \zeta_{2}\right)^{6}, \\
\eta_{1} & =\zeta_{6} \zeta_{3} \zeta_{2}^{-1} \zeta_{3}^{2} \zeta_{6}^{-1} \zeta_{3}^{-2} \zeta_{2} \zeta_{3}^{-1}, \\
\eta_{2} & =\zeta_{6} \zeta_{3}^{2} \zeta_{2}^{-1} \zeta_{3} \zeta_{6}^{-1} \zeta_{3}^{-1} \zeta_{2} \zeta_{3}^{-2} .
\end{aligned}
$$

Then we can check that $J\left(\eta_{1}\right)=J\left(\eta_{2}\right)$ but $\eta_{1}$ and $\eta_{2}$ are not conjugate in $\Delta_{g}$, because

$$
\left\{A \in \widetilde{\mathrm{GL}}_{5}(\mathbb{Z}) \mid A \eta_{1}=\eta_{2} A\right\} \cap \mathrm{Sp}(q, 2)=\varnothing .
$$

\section{TABLES}

\section{A. Conjugacy Classes for $g=2$}

Table 1 gives the word expression, Meyer's function, Sp-representation, and modified Jones representation for all conjugacy classes up to word length 4 . In the first column we have elements of $\Delta_{2}$. As an example of how to read the word expression, $\{1,-2,3\}$ means $\zeta_{1} \zeta_{2}^{-1} \zeta_{3}$.

The characteristic function $I(\zeta)$ of the Sp-representation is defined by

$$
I(\zeta)=\operatorname{det}\left(z E_{4}-\operatorname{Sp}(\zeta)\right)
$$

for $\zeta \in \Delta_{2}=\mathcal{M}_{2}$. It is easy to show that

$$
I(\zeta)=y^{4}+i_{1}(\zeta) y^{3}+i_{2}(\zeta) y^{2}+i_{1}(\zeta) y+1 .
$$

We have $i(\zeta)$ in our table.
The characteristic function $J(\zeta)$ of the Jones representation $\rho_{2}$ is defined by

$$
J(\zeta)=\operatorname{det}\left(y E_{5}-\rho_{2}(\zeta)\right)
$$

for $\zeta \in \Delta_{2}=\mathcal{M}_{2}$. Here $\rho_{2}$ was already given by Jones [1987]. From Corollary 4.4,

$$
\begin{aligned}
J(\zeta)=y^{5} & +\varepsilon j_{1}(q) y^{4}+j_{2}(q) y^{3} \\
& +\varepsilon j_{2}\left(q^{-1}\right) y^{2}+j_{1}\left(q^{-1}\right) y+\varepsilon,
\end{aligned}
$$

where $\varepsilon=1$ if the length of the word is even and $\varepsilon=-1$ if the length of the word is odd. In the table, $n\left(b_{0}, b_{1}, b_{2}, \ldots\right)$ means $q^{n}\left(b_{0}+b_{1} q+b_{2} q^{2}+\cdots\right)$. In the fourth column, we have the Thurston type of each conjugacy classes. We can get the invariant train track (and hence Thurston type) for all of elements of $\mathcal{M}_{g}$ using the algorithm from [1992].

In the table we omit the data for inverses. For example, if we have the data of $\zeta_{1}^{3}$ we omit those of $\zeta_{1}^{-3}$. The data for inverse elements are obtained via the formulas

$$
\begin{aligned}
\varphi_{2}\left(\zeta^{-1}\right) & =-\varphi_{2}(\zeta), \\
i_{1}\left(\zeta^{-1}\right) & =i_{1}(\zeta), \\
i_{2}\left(\zeta^{-1}\right) & =i_{2}(\zeta), \\
j_{1}\left(\zeta^{-1}\right)(q) & =j_{1}(\zeta)\left(q^{-1}\right), \\
j_{2}\left(\zeta^{-1}\right)(q) & =j_{2}(\zeta)\left(q^{-1}\right) .
\end{aligned}
$$

\begin{tabular}{|lrrrlll|}
\hline$\zeta$ & $\varphi_{2}$ & $i_{1}$ & $i_{2}$ & type & $j_{1}(q)$ & $j_{2}(q)$ \\
\hline$\{1\}$ & $3 / 5$ & -4 & 6 & reducible & $-2 / 5(-3,2)$ & $-4 / 5(3,-6,1)$ \\
$\{1,1\}$ & $1 / 5$ & -4 & 6 & reducible & $-4 / 5(-3,0,-2)$ & $-8 / 5(3,0,6,0,1)$ \\
$\{1,2\}$ & $6 / 5$ & -3 & 4 & reducible & $-4 / 5(-1,2)$ & $-3 / 5(-2,3)$ \\
$\{1,-2\}$ & 0 & -5 & 8 & reducible & $-1(2,-3,2)$ & $-2(1,-4,7,-4,1)$ \\
$\{1,3\}$ & $6 / 5$ & -4 & 6 & reducible & $-4 / 5(-2,2,-1)$ & $-8 / 5(1,-4,3,-2)$ \\
$\{1,-3\}$ & 0 & -4 & 6 & reducible & $-1(1,-3,1)$ & $-1(-3,4,-3)$ \\
$\{1,1,1\}$ & $-1 / 5$ & -4 & 6 & reducible & $-6 / 5(-3,0,0,2)$ & $-12 / 5(3,0,0,-6,0,0,1)$ \\
$\{1,1,2\}$ & $4 / 5$ & -2 & 2 & reducible & $-6 / 5(-1)$ & $3 / 5(-2)$ \\
$\{1,1,-2\}$ & $-2 / 5$ & -6 & 10 & reducible & $-7 / 5(2,-3,2,-2)$ & $-14 / 5(1,-4,5,-8,5,-2,1)$ \\
$\{1,1,3\}$ & $4 / 5$ & -4 & 6 & reducible & $-6 / 5(-2,1,-1,1)$ & $-12 / 5(1,-2,2,-3,1,-1)$ \\
$\{1,1,-3\}$ & $-2 / 5$ & -4 & 6 & reducible & $-7 / 5(1,-2,1,-1)$ & $-9 / 5(-2,2,-3,2,-1)$ \\
$\{1,2,3\}$ & $9 / 5$ & -2 & 2 & reducible & $-1 / 5(1)$ & $-2 / 5(1,-1)$ \\
$\{1,2,-3\}$ & $3 / 5$ & -4 & 6 & reducible & $-7 / 5(1,-2,2)$ & $-9 / 5(-1,3,-4,2)$ \\
$\{1,2,4\}$ & $9 / 5$ & -3 & 4 & reducible & $-6 / 5(-1,1,-1)$ & $-7 / 5(-1,2,-1,1)$ \\
$\{1,2,-4\}$ & $3 / 5$ & -3 & 4 & reducible & $-2 / 5(-2,1)$ & $-4 / 5(2,-2,1)$ \\
$\{1,-2,3\}$ & $3 / 5$ & -6 & 10 & reducible & $-7 / 5(2,-3,3,-1)$ & $-14 / 5(1,-3,7,-8,5,-2)$ \\
\hline
\end{tabular}

TABLE 1. Word expression, Meyer's function, Sp-representation, and modified Jones representation for conjugacy classes up to word length 4 , when $g=2$. See Section 5 A. 


\begin{tabular}{|c|c|c|c|c|c|c|}
\hline$\zeta$ & $\varphi_{2}$ & $i_{1}$ & $i_{2}$ & type & $j_{1}(q)$ & $j_{2}(q)$ \\
\hline$\{1,-2,4\}$ & $3 / 5$ & -5 & 8 & reducible & $-7 / 5(1,-3,2,-1)$ & $-9 / 5(-2,5,-5,4,-1)$ \\
\hline$\{1,3,5\}$ & $4 / 5$ & -4 & 6 & reducible & $-6 / 5(-1,3,0,1)$ & $-7 / 5(-3,3,-1,3)$ \\
\hline$\{1,3,-5\}$ & $-2 / 5$ & -4 & 6 & reducible & $-7 / 5(1,-1,3)$ & $-9 / 5(-1,3,-3,3)$ \\
\hline$\{1,1,1,1\}$ & $-3 / 5$ & -4 & 6 & reducible & $-8 / 5(-3,0,0,0,-2)$ & $-16 / 5(3,0,0,0,6,0,0,0,1)$ \\
\hline$\{1,1,1,2\}$ & $2 / 5$ & -1 & 0 & reducible & $-8 / 5(-1,0,2)$ & $-6 / 5(-2,0,3)$ \\
\hline$\{1,1,1,-2\}$ & $-4 / 5$ & -7 & 12 & reducible & $-9 / 5(2,-3,2,-2,2)$ & $-18 / 5(1,-4,5,-6,9,-6,3,-2,1)$ \\
\hline$\{1,1,1,3\}$ & $2 / 5$ & -4 & 6 & reducible & $-8 / 5(-2,1,0,1,-1)$ & $-16 / 5(1,-2,0,-2,3,-1,0,-1)$ \\
\hline$\{1,1,1,-3\}$ & $-4 / 5$ & -4 & 6 & reducible & $-9 / 5(1,-2,0,-1,1)$ & $-13 / 5(-2,1,-1,3,-2,0,-1)$ \\
\hline$\{1,1,2,2\}$ & $2 / 5$ & 0 & -2 & reducible & $-8 / 5(-1,-2,0,-2)$ & $-11 / 5(2,1,2,4,0,1)$ \\
\hline$\{1,1,2,3\}$ & $7 / 5$ & -1 & 0 & reducible & $2 / 5(1)$ & $4 / 5(1)$ \\
\hline$\{1,1,2,-3\}$ & $1 / 5$ & -3 & 4 & reducible & $-9 / 5(1,-1,1)$ & $-8 / 5(1,-1,2,-1)$ \\
\hline$\{1,1,2,4\}$ & $7 / 5$ & -2 & 2 & reducible & $-8 / 5(-1)$ & $-1 / 5(-1,0,-1)$ \\
\hline$\{1,1,2,-4\}$ & $1 / 5$ & -2 & 2 & reducible & $-4 / 5(-1)$ & $-3 / 5(-1,0,-1)$ \\
\hline$\{1,1,-2,-2\}$ & 0 & -8 & 14 & reducible & $-2(-2,2,-5,2,-2)$ & $-4(1,-2,7,-8,14,-8,7,-2,1)$ \\
\hline$\{1,1,-2,3\}$ & $1 / 5$ & -7 & 12 & reducible & $-9 / 5(2,-3,3,-2,1)$ & $-18 / 5(1,-3,6,-8,9,-6,3,-1)$ \\
\hline$\{1,1,-2,-3\}$ & -1 & -5 & 8 & reducible & $-1(2,-2,2,-1)$ & $-2(2,-3,5,-4,2,-1)$ \\
\hline$\{1,1,-2,4\}$ & $1 / 5$ & -6 & 10 & reducible & $-9 / 5(1,-3,2,-2,1)$ & $-13 / 5(-2,4,-6,6,-5,2,-1)$ \\
\hline$\{1,1,-2,-4\}$ & -1 & -6 & 10 & reducible & $-2(-1,2,-3,2,-1)$ & $-3(-1,3,-6,6,-6,3,-1)$ \\
\hline$\{1,1,3,3\}$ & $2 / 5$ & -4 & 6 & reducible & $-8 / 5(-2,0,-2,0,-1)$ & $-16 / 5(1,0,4,0,3,0,2)$ \\
\hline$\{1,1,3,4\}$ & $7 / 5$ & -3 & 4 & reducible & $-8 / 5(-1,1,0,1)$ & $-11 / 5(-1,1,-1,1,0,1)$ \\
\hline$\{1,1,3,-4\}$ & $1 / 5$ & -5 & 8 & reducible & $-9 / 5(1,-2,2,-1,1)$ & $-13 / 5(-1,3,-4,4,-3,2)$ \\
\hline$\{1,1,3,5\}$ & $2 / 5$ & -4 & 6 & reducible & $-8 / 5(-1,2,-1,0,-1)$ & $-11 / 5(-2,2,-2,1,-2,1)$ \\
\hline$\{1,1,3,-5\}$ & $-4 / 5$ & -4 & 6 & reducible & $-9 / 5(1,-1,1,-2)$ & $-13 / 5(-1,1,-3,2,-2,1)$ \\
\hline$\{1,1,-3,-3\}$ & 0 & -4 & 6 & reducible & $-2(-1,0,-3,0,-1)$ & $-2(3,0,4,0,3)$ \\
\hline$\{1,1,-3,-4\}$ & -1 & -3 & 4 & reducible & $-1(1,-1,1)$ & $-2(1,-1,1,-1,1)$ \\
\hline$\{1,1,-3,-5\}$ & 0 & -4 & 6 & reducible & $-1(2,-2,0,-1)$ & $-2(1,-4,1,-2,2)$ \\
\hline$\{1,2,2,3\}$ & $7 / 5$ & 0 & -2 & reducible & $-3 / 5(-1,1,-1)$ & $-6 / 5(-1,-1,1,-1)$ \\
\hline$\{1,2,2,-3\}$ & $1 / 5$ & -4 & 6 & reducible & $-9 / 5(1,-2,1,-1)$ & $-13 / 5(-1,1,-3,3,-2)$ \\
\hline$\{1,2,3,4\}$ & $12 / 5$ & -1 & 1 & period 5 & $0(0)$ & $0(0)$ \\
\hline$\{1,2,3,-4\}$ & $6 / 5$ & -3 & 3 & pseudo-An. & $-4 / 5(-1,1)$ & $-8 / 5(1,-2,2,-1)$ \\
\hline$\{1,2,3,5\}$ & $7 / 5$ & -2 & 2 & reducible & $-3 / 5(1)$ & $-6 / 5(1,0,0,-1)$ \\
\hline$\{1,2,3,-5\}$ & $1 / 5$ & -2 & 2 & reducible & $1 / 5(1)$ & $-3 / 5(-1,1)$ \\
\hline$\{1,2,-3,4\}$ & $6 / 5$ & -5 & 7 & pseudo-An. & $-9 / 5(1,-2,2,-1)$ & $-13 / 5(-1,3,-5,5,-3,1)$ \\
\hline$\{1,2,-3,-4\}$ & 0 & -3 & 5 & pseudo-An. & $-1(1,-2,1)$ & $-1(-1,2,-1)$ \\
\hline$\{1,2,-3,5\}$ & $1 / 5$ & -4 & 6 & reducible & $-9 / 5(1,-1,2,-1)$ & $-8 / 5(2,-3,3,-2)$ \\
\hline$\{1,2,-3,-5\}$ & 1 & -4 & 6 & reducible & $-1(2,-2,1)$ & $-2(1,-3,3,-2,1)$ \\
\hline$\{1,2,4,5\}$ & $12 / 5$ & -2 & 3 & reducible & $-8 / 5(-1,0,-1)$ & $-6 / 5(1)$ \\
\hline$\{1,2,4,-5\}$ & $6 / 5$ & -4 & 5 & reducible & $-4 / 5(-2,1,-1)$ & $-8 / 5(2,-3,3,-2,1)$ \\
\hline$\{1,2,-4,-5\}$ & 0 & -2 & 3 & period 3 & $0(-2)$ & $0(1)$ \\
\hline$\{1,-2,1,-2\}$ & 0 & -9 & 16 & reducible & $-2(-2,4,-3,4,-2)$ & $-4(1,-4,8,-12,15,-12,8,-4,1)$ \\
\hline$\{1,-2,-2,3\}$ & 1 & -8 & 14 & reducible & $-2(-2,3,-4,3,-1)$ & $-4(1,-3,6,-11,12,-10,5,-2)$ \\
\hline$\{1,-2,3,-2\}$ & 1 & -9 & 16 & reducible & $-2(-2,4,-4,4,-1)$ & $-4(1,-4,8,-14,16,-12,8,-2)$ \\
\hline$\{1,-2,3,-4\}$ & 0 & -7 & 13 & pseudo-An. & $-2(-1,3,-4,3,-1)$ & $-3(-1,4,-8,10,-8,4,-1)$ \\
\hline$\{1,-2,3,5\}$ & $1 / 5$ & -6 & 10 & reducible & $-9 / 5(1,-3,3,-1,1)$ & $-13 / 5(-1,5,-7,6,-5,2)$ \\
\hline$\{1,-2,3,-5\}$ & -1 & -6 & 10 & reducible & $-2(-1,2,-3,3)$ & $-3(-1,3,-6,7,-6,3)$ \\
\hline$\{1,-2,-3,4\}$ & 0 & -5 & 9 & pseudo-An. & $-1(2,-3,2,-1)$ & $-2(1,-4,5,-4,2)$ \\
\hline$\{1,-2,4,-5\}$ & 0 & -6 & 11 & reducible & $-2(-1,2,-4,2,-1)$ & $-2(3,-6,7,-6,3)$ \\
\hline
\end{tabular}

TABLE 1 (continued) 


\section{B. The Torelli Group}

The Torelli group $\mathcal{J}_{g} \subset \mathcal{M}_{g}$ is the kernel of the Sphomomorphism. When $g=2$, it is generated by Dehn twists of all separating simple closed curves:

$$
\mathcal{J}_{2}=\left\langle C(\kappa) \zeta_{6} \mid \kappa \in \mathcal{M}_{g}\right\rangle,
$$

where $\zeta_{6}=\left(\zeta_{1} \zeta_{2}\right)^{6}$ is the Dehn twist of a standard separating simple closed curve $D_{0}$ as in the figure.

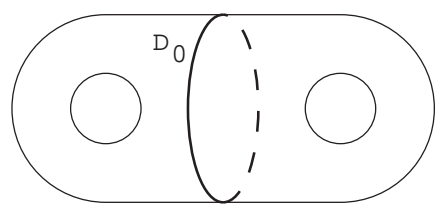

It is known that $\mathcal{J}_{2}$ is infinitely generated. The following relations on $\zeta_{6}$ hold:

Lemma 5.1. (1) $\zeta_{6}=\left(\zeta_{4} \zeta_{5}\right)^{6}$.

(2) $C\left(\eta_{1,5}\right)\left(\zeta_{6}\right)=\zeta_{6}, C\left(\xi^{3}\right)\left(\zeta_{6}\right)=\zeta_{6}$.

(3) $\zeta_{i} \zeta_{6}=\zeta_{6} \zeta_{i}$ for $i=1,2,4,5$.

Proof. From the Birman-Hilden relations,

$$
\begin{aligned}
\sigma \zeta_{1}^{-1} \zeta_{2}^{-1} \zeta_{3}^{-1} \zeta_{4}^{-1} \zeta_{5}^{-1} & =\zeta_{1} \zeta_{2} \zeta_{3} \zeta_{4} \zeta_{5}, \\
\sigma^{3}\left(\zeta_{1}^{-1} \zeta_{2}^{-1} \zeta_{3}^{-1} \zeta_{4}^{-1} \zeta_{5}^{-1}\right)^{3}\left(\zeta_{1} \zeta_{2} \zeta_{3} \zeta_{4} \zeta_{5}\right)^{3} & =1, \\
\left(\zeta_{1} \zeta_{2}\right)^{3}\left(\zeta_{4}^{-1} \zeta_{5}^{-1}\right)^{3} & =\sigma, \\
\left(\zeta_{1} \zeta_{2}\right)^{6}=\left(\zeta_{5} \zeta_{4}\right)^{6} & =\left(\zeta_{4} \zeta_{5}\right)^{6} .
\end{aligned}
$$

Next, from Lemma 2.1(1),(3),

$$
\begin{array}{ll}
C\left(\eta_{1,5}\right)\left(\zeta_{1}\right)=\zeta_{5}, & C\left(\xi^{3}\right)\left(\zeta_{1}\right)=\zeta_{4}, \\
C\left(\eta_{1,5}\right)\left(\zeta_{2}\right)=\zeta_{4}, & C\left(\xi^{3}\right)\left(\zeta_{2}\right)=\zeta_{5} .
\end{array}
$$

The conclusions follow.

There is exactly one conjugacy class of word length 1 in the Torelli group $\mathcal{J}_{2}$, namely $\zeta_{6}$. Conjugacy classes of word length 2 in $\mathcal{J}_{2}$ are given by

$$
D_{ \pm}(\kappa):=\kappa \zeta_{6} \kappa^{-1} \zeta_{6}^{ \pm 1}
$$

for any $\kappa \in \mathcal{M}_{2}$. Table 2 shows $\zeta_{6}$ and $D_{ \pm}(\kappa)$ for $\kappa$ with at most word length 4 . From this table we see that the characteristic function of the Jones representation cannot distinguish all conjugacy classes of $\mathcal{M}_{2}$. We also give some lemmas on $D_{ \pm}(\kappa)$, which follow easily from Lemma 5.1. As before, $\sim$ means conjugacy in $\mathcal{M}_{g}$.

Lemma 5.2. (1) For any $\kappa, D_{ \pm}\left(\kappa \eta_{1,5}\right) \sim D_{ \pm}\left(\eta_{1,5} \kappa\right) \sim$ $D_{ \pm}(\kappa)$.

(2) For any $\kappa, D_{ \pm}\left(\kappa \xi^{3}\right) \sim D_{ \pm}\left(\xi^{3} \kappa\right) \sim D_{ \pm}(\kappa)$.
(3) For any $\kappa$ and $i=1,2,4,5$,

$$
D_{ \pm}\left(\kappa \zeta_{i}\right) \sim D_{ \pm}\left(\zeta_{i} \kappa\right) \sim D_{ \pm}(\kappa) .
$$

(4) For any $\kappa, D_{+}\left(\kappa^{-1}\right) \sim D_{+}(\kappa)$ and $D_{-}\left(\kappa^{-1}\right) \sim$ $\left(D_{-}(\kappa)\right)^{-1}$.

We give some nontrivial relations. Let $D_{ \pm}\left(b_{1}, b_{2}, \ldots\right)$ denote $D_{ \pm}\left(\left\{b_{1}, b_{2}, \ldots\right\}\right)$.

Lemma 5.3. (1) $D_{ \pm}(3,2,4,3) \sim D_{ \pm}(-3,-2,4,3) \sim$ $D_{ \pm}(-3)$.

(2) $D_{ \pm}(3,2,2,3) \sim D_{ \pm}(-3,-3)$.

(3) $D_{ \pm}(3,2,4,-3) \sim D_{ \pm}(3,2,-4,3) \sim D_{ \pm}(3,-2,4,3)$ $\sim D_{ \pm}(-3,2,4,3)$.

Proof. (1) If $\xi^{\prime}=\zeta_{5} \zeta_{4} \zeta_{3} \zeta_{2} \zeta_{1}$ then $D_{ \pm}(\kappa) \sim D_{ \pm}\left(\xi^{\prime 3} \kappa\right)$. Using Lemma 5.1,

$$
\begin{aligned}
D_{ \pm}(3, & 2,4,3) \\
\sim & D_{ \pm}\left(\xi^{\prime-3} \zeta_{3} \zeta_{2} \zeta_{4} \zeta_{3}\right) \\
= & D_{ \pm}(-1,-2,-3,-4,-5,-1,-2,-3 \\
& \quad-4,-5,-1,-2,-3,-4,-5,3,2,4,3) \\
= & D_{ \pm}(-1,-2,-1,-2,-1,-2,-3,-2,-1,-4,-5) \\
\sim & D_{ \pm}(-3) .
\end{aligned}
$$

Because $\xi \xi^{\prime-2}=\xi^{3}$,

$$
\begin{aligned}
D_{ \pm}(-3 & ,-2,4,3) \\
\sim & D_{ \pm}\left(\xi \xi^{\prime-2} \zeta_{3} \zeta_{2} \zeta_{4} \zeta_{3}\right) \\
= & D_{ \pm}(1,2,3,4,5,-1,-2,-3,-4,-5 \\
& \quad-1,-2,-3,-4,-5,-3,-2,4,3) \\
= & D_{ \pm}(1,2,-1,-2,-1,-2,-3,-2,1,-4,-5) \\
\sim & D_{ \pm}(-3) .
\end{aligned}
$$

(2) We have

$$
\begin{aligned}
C\left(\xi^{-3}\right)\left(\zeta_{3}\right) & =C\left(\xi^{-1} \xi^{2}\right)\left(\zeta_{3}\right) \\
& =\{-1,-2,-3,-4,-5,-4,-3,-2,-1\} .
\end{aligned}
$$

Therefore

$D_{ \pm}(3,2,2,3)$

$$
\begin{aligned}
& \sim D_{ \pm}\left(C\left(\xi^{\prime-3}\right)\left(\zeta_{3} \zeta_{2}^{2} \zeta_{3}\right)\right) \\
= & D_{ \pm}(-1,-2,-3,-4,-5,-4,-3,-2,-1,5 \\
& \quad 5,-1,-2,-3,-4,-5,-4,-3,-2,-1) \\
= & D_{ \pm}(-1,-2,-3,-3,-2,-1) \\
\sim & D_{ \pm}(-3,-3) .
\end{aligned}
$$




\begin{tabular}{|c|c|c|c|c|}
\hline$\zeta$ & $\varphi_{2}$ & $i$ & type & $\begin{array}{l}j_{1}(q) \\
j_{2}(q)\end{array}$ \\
\hline$\{6\}$ & $-4 / 5$ & -2 & reducible & $\begin{array}{l}-24 / 5(-1,0,0,0,0,0,-4) \\
-18 / 5(4,0,0,0,0,0,6)\end{array}$ \\
\hline$D_{+}(3)$ & $-8 / 5$ & -2 & reducible & $\begin{array}{l}-38 / 5(-1,2,-1,-2,2,-2,-1,2,-1,0,-3) \\
-36 / 5(1,0,3,-6,3,6,-6,6,3,-6,3,0,3)\end{array}$ \\
\hline$D_{+}(3,3)$ & $-8 / 5$ & -2 & reducible & $\begin{array}{l}-53 / 5(-1,1,0,-4,6,-2,-5,8,-5,-2,6,-4,0,-2,-1) \\
-41 / 5(3,-2,0,12,-18,6,15,-24,15,6,-18,12,0,0,3)\end{array}$ \\
\hline$D_{+}(3,-2,3)$ & $-8 / 5$ & -2 & reducible & $\begin{array}{l}-58 / 5(1,-3,3,2,-11,14,-4,-13,20,-13,-4,14,-11,2,0,-3,1) \\
-46 / 5(-3,9,-8,-6,33,-42,12,39,-60,39,12,-42,33,-6,-6,9,-3)\end{array}$ \\
\hline$D_{+}(3,3,3)$ & $-8 / 5$ & -2 & reducible & $\begin{array}{l}-58 / 5(1,-2,1,2,-7,8,-2,-8,12,-8,-2,8,-7,2,-2,-2,1) \\
-46 / 5(-3,6,-2,-6,21,-24,6,24,-36,24,6,-24,21,-6,0,6,-3)\end{array}$ \\
\hline$D_{+}(3,-2,-2,3)$ & $-8 / 5$ & -2 & reducible & $\begin{array}{l}-63 / 5(-1,3,-5,2,7,-18,19,-3,-20,30,-20,-3,19,-18,7,-1,-5,3,-1) \\
-51 / 5(3,-9,15,-5,-21,54,-57,9,60,-90,60,9,-57,54,-21,-3,15,-9,3)\end{array}$ \\
\hline$D_{+}(3,-2,3,3)$ & $-8 / 5$ & -2 & reducible & $\begin{array}{l}-63 / 5(-1,4,-7,4,9,-25,27,-5,-28,42,-28,-5,27,-25,9,1,-7,4,-1) \\
-51 / 5(3,-12,21,-11,-27,75,-81,15,84,-126,84,15,-81,75,-27,-9,21,-12,3)\end{array}$ \\
\hline$D_{+}(3,-2,4,3)$ & $-8 / 5$ & -2 & pseudo-An. & $\begin{array}{l}-48 / 5(-1,4,-8,8,0,-12,16,-12,0,8,-8,4,-4) \\
-36 / 5(4,-12,24,-24,0,36,-48,36,0,-24,24,-12,6)\end{array}$ \\
\hline$D_{+}(3,-2,-4,3)$ & $-8 / 5$ & -2 & pseudo-An. & $\begin{array}{l}-63 / 5(-1,4,-7,4,9,-25,27,-5,-28,42,-28,-5,27,-25,9,1,-7,4,-1) \\
-51 / 5(3,-12,21,-11,-27,75,-81,15,84,-126,84,15,-81,75,-27,-9,21,-12,3)\end{array}$ \\
\hline$D_{+}(3,3,-2,3)$ & $-8 / 5$ & -2 & reducible & $\begin{array}{l}-63 / 5(-1,4,-7,4,9,-25,27,-5,-28,42,-28,-5,27,-25,9,1,-7,4,-1) \\
-51 / 5(3,-12,21,-11,-27,75,-81,15,84,-126,84,15,-81,75,-27,-9,21,-12,3)\end{array}$ \\
\hline$D_{+}(3,3,3,3)$ & $-8 / 5$ & -2 & reducible & $\begin{array}{l}-63 / 5(-1,2,-2,-1,5,-8,7,0,-9,12,-9,0,7,-8,5,-4,-2,2,-1) \\
-51 / 5(3,-6,6,4,-15,24,-21,0,27,-36,27,0,-21,24,-15,6,6,-6,3)\end{array}$ \\
\hline$D_{-}(3)$ & 0 & -2 & reducible & $\begin{array}{l}-6(-1,0,1,-2,1,2,-7,2,1,-2,1,0,-1) \\
-6(3,0,-3,6,-3,-6,16,-6,-3,6,-3,0,3)\end{array}$ \\
\hline$D_{-}(3,3)$ & 0 & -2 & reducible & $\begin{array}{l}-7(1,-2,0,4,-6,2,5,-13,5,2,-6,4,0,-2,1) \\
-7(-3,6,0,-12,18,-6,-15,34,-15,-6,18,-12,0,6,-3)\end{array}$ \\
\hline$D_{-}(3,-2,3)$ & 0 & -2 & reducible & $\begin{array}{l}-8(-1,3,-4,-2,11,-14,4,13,-25,13,4,-14,11,-2,-4,3,-1) \\
-8(3,-9,12,6,-33,42,-12,-39,70,-39,-12,42,-33,6,12,-9,3)\end{array}$ \\
\hline$D_{-}(3,3,3)$ & 0 & -2 & reducible & $\begin{array}{l}-8(-1,2,-2,-2,7,-8,2,8,-17,8,2,-8,7,-2,-2,2,-1) \\
-8(3,-6,6,6,-21,24,-6,-24,46,-24,-6,24,-21,6,6,-6,3)\end{array}$ \\
\hline$D_{-}(3,-2,-2,3)$ & 0 & -2 & reducible & $\begin{array}{l}-9(1,-3,5,-3,-7,18,-19,3,20,-35,20,3,-19,18,-7,-3,5,-3,1) \\
-9(-3,9,-15,9,21,-54,57,-9,-60,100,-60,-9,57,-54,21,9,-15,9,-3)\end{array}$ \\
\hline$D_{-}(3,-2,3,3)$ & 0 & -2 & reducible & $\begin{array}{l}-9(1,-4,7,-5,-9,25,-27,5,28,-47,28,5,-27,25,-9,-5,7,-4,1) \\
-9(-3,12,-21,15,27,-75,81,-15,-84,136,-84,-15,81,-75,27,15,-21,12,-3)\end{array}$ \\
\hline$D_{-}(3,-2,4,3)$ & 0 & -2 & pseudo-An. & $\begin{array}{l}-5(-4,8,-8,0,12,-21,12,0,-8,8,-4) \\
-5(12,-24,24,0,-36,58,-36,0,24,-24,12)\end{array}$ \\
\hline$D_{-}(3,-2,-4,3)$ & 0 & -2 & pseudo-An. & $\begin{array}{l}-9(1,-4,7,-5,-9,25,-27,5,28,-47,28,5,-27,25,-9,-5,7,-4,1) \\
-9(-3,12,-21,15,27,-75,81,-15,-84,136,-84,-15,81,-75,27,15,-21,12,-3)\end{array}$ \\
\hline$D_{-}(3,3,-2,3)$ & 0 & -2 & reducible & $\begin{array}{l}-9(1,-4,7,-5,-9,25,-27,5,28,-47,28,5,-27,25,-9,-5,7,-4,1) \\
-9(-3,12,-21,15,27,-75,81,-15,-84,136,-84,-15,81,-75,27,15,-21,12,-3)\end{array}$ \\
\hline$D_{-}(3,3,3,3)$ & 0 & -2 & reducible & $\begin{array}{l}-9(1,-2,2,0,-5,8,-7,0,9,-17,9,0,-7,8,-5,0,2,-2,1) \\
-9(-3,6,-6,0,15,-24,21,0,-27,46,-27,0,21,-24,15,0,-6,6,-3)\end{array}$ \\
\hline
\end{tabular}

TABLE 2. Data for $\zeta_{6}$ and $D_{ \pm}(\kappa)$, for $\kappa$ of word length at most 4 .

(3) We have

$$
\begin{aligned}
& D_{ \pm}(3,2,4,-3)=D_{ \pm}(3,2,-3,3,4,-3) \\
& =D_{ \pm}(-2,3,2,-4,3,4) \sim D_{ \pm}(3,2,-4,3), \\
& D_{ \pm}(3,2,-4,3)=D_{ \pm}(3,-4,2,3) \\
& \sim D_{ \pm}\left(C\left(\eta_{1,5}\right)(\{3,-4,2,3\})\right)=D_{ \pm}(3,-2,4,3), \\
& D_{ \pm}(3,-2,4,3) \sim D_{ \pm}(2,3,-2,4,3,-4) \\
& =D_{ \pm}(-3,2,3,-3,4,3)=D_{ \pm}(-3,2,4,3) .
\end{aligned}
$$

Lemma 5.4. (1) $D_{ \pm}(3,-2,3,3) \nsim D_{ \pm}(3,3,-2,3)$.

(2) $D_{ \pm}(3,-2,3,3) \nsim D_{ \pm}(3,-2,-4,3)$.

(3) $D_{ \pm}(3,3,-2,3) \not D_{ \pm}(3,-2,-4,3)$.
Proof. (1) Let

$$
\eta_{1}=D_{+}(3,-2,3,3), \quad \eta_{2}=D_{+}(3,3,-2,3) .
$$

A short calculation shows that the set

$$
\left\{A \in \widetilde{\mathrm{GL}_{5}}(\mathbb{Z}) \mid A \eta_{1}=\eta_{2} A\right\} \cap \operatorname{Sp}(q, 2)
$$

is empty. Thus $\eta_{1}$ and $\eta_{2}$ are not conjugate.

(2), (3) Using the second author's software (see section on Electronic Availability at the end of this paper), we check that the stretch factors of the invariant train tracks of $D_{+}(3,-2,3,3), D_{+}(3,-2,-4,3)$, $D_{-}(3,-2,3,3)$, and $D_{-}(3,-2,-4,3)$ are approximately 398, 254, 402, and 258, respectively. Since 
the stretch factor is an invariant of conjugation, none of these $D$ 's can be conjugate.

\section{C. Conjugacy Classes for $\mathrm{g}=3$}

Finally we discuss the Jones representation in the case $g=3$. We consider a $2 \times 4$ rectangular Young diagram, obtaining the $\mathrm{W}$-graph shown on page 385 , bottom right. Moreover $\rho_{3}\left(\zeta_{1}\right)$ equals $q^{-5 / 14}$ times

$\left(\begin{array}{rrrrrrrrrrrrrr}q & 0 & 0 & 0 & 0 & 0 & 0 & 0 & 0 & 0 & 0 & 0 & 0 & 0 \\ q & -1 & 0 & 0 & 0 & 0 & 0 & 0 & 0 & 0 & 0 & 0 & 0 & 0 \\ 0 & 0 & q & 0 & 0 & 0 & 0 & 0 & 0 & 0 & 0 & 0 & 0 & 0 \\ q & 0 & 0 & -1 & 0 & 0 & 0 & 0 & 0 & 0 & 0 & 0 & 0 & 0 \\ 0 & 0 & 0 & 0 & q & 0 & 0 & 0 & 0 & 0 & 0 & 0 & 0 & 0 \\ 0 & 0 & 0 & 0 & 0 & q & 0 & 0 & 0 & 0 & 0 & 0 & 0 & 0 \\ q & 0 & 0 & 0 & 0 & 0 & -1 & 0 & 0 & 0 & 0 & 0 & 0 & 0 \\ 0 & 0 & 0 & 0 & 0 & 1 & 0 & -1 & 0 & 0 & 0 & 0 & 0 & 0 \\ 0 & 0 & 0 & 0 & 1 & 0 & 0 & 0 & -1 & 0 & 0 & 0 & 0 & 0 \\ 0 & 0 & 0 & 0 & 0 & 0 & 0 & 0 & 0 & q & 0 & 0 & 0 & 0 \\ 0 & 0 & 1 & 0 & 0 & 0 & 0 & 0 & 0 & 0 & -1 & 0 & 0 & 0 \\ 0 & 0 & 0 & 0 & 1 & 0 & 0 & 0 & 0 & 0 & 0 & -1 & 0 & 0 \\ 0 & 0 & 0 & 0 & 0 & 1 & 0 & 0 & 0 & 0 & 0 & 0 & -1 & 0 \\ 0 & 0 & 0 & 0 & 0 & 0 & 0 & 0 & 0 & q & 0 & 0 & 0 & -1\end{array}\right)$

and $\rho_{3}\left(\zeta_{1} \zeta_{2} \zeta_{3} \zeta_{4} \zeta_{5} \zeta_{6} \zeta_{7}\right)$ equals

$$
\left(\begin{array}{cccccccccccccc}
0 & 0 & 0 & 0 & 0 & 0 & 0 & 0 & 0 & 0 & 0 & 0 & 0 & q_{2} \\
0 & 0 & 0 & 0 & 0 & 0 & 0 & 0 & 0 & 0 & 0 & 0 & q_{1} & 0 \\
0 & 0 & 0 & 0 & 0 & 0 & 0 & 0 & 0 & 0 & 0 & q_{1} & 0 & 0 \\
0 & 0 & 0 & 0 & 0 & 0 & 0 & 0 & q_{1} & 0 & 0 & 0 & 0 & 0 \\
0 & 0 & 0 & 0 & 0 & 0 & 0 & q_{1} & 0 & 0 & 0 & 0 & 0 & 0 \\
0 & 0 & 0 & 0 & 0 & 0 & 0 & 0 & 0 & 0 & q_{1} & 0 & 0 & 0 \\
0 & 0 & 0 & 0 & 0 & 0 & 0 & 0 & 0 & q_{1} & 0 & 0 & 0 & 0 \\
0 & 0 & 0 & 0 & 0 & 0 & q_{2} & 0 & 0 & 0 & 0 & 0 & 0 & 0 \\
0 & 0 & 0 & 0 & 0 & q_{2} & 0 & 0 & 0 & 0 & 0 & 0 & 0 & 0 \\
0 & 0 & 0 & q_{2} & 0 & 0 & 0 & 0 & 0 & 0 & 0 & 0 & 0 & 0 \\
0 & 0 & 0 & 0 & q_{2} & 0 & 0 & 0 & 0 & 0 & 0 & 0 & 0 & 0 \\
0 & q_{2} & 0 & 0 & 0 & 0 & 0 & 0 & 0 & 0 & 0 & 0 & 0 & 0 \\
0 & 0 & q_{2} & 0 & 0 & 0 & 0 & 0 & 0 & 0 & 0 & 0 & 0 & 0 \\
q_{1} & 0 & 0 & 0 & 0 & 0 & 0 & 0 & 0 & 0 & 0 & 0 & 0 & 0
\end{array}\right),
$$

where we have put $q_{1}=-q^{1 / 2}$ and $q_{2}=-q^{-1 / 2}$.

Table 3 shows the results obtained.

\section{ELECTRONIC AVAILABILITY}

The software used to compute the tables given in this paper is available at http://www.is.titech.ac.jp/ -takasawa/MCG/index.html. Software implementing the train track algorithm can be found at the same address.

\section{ACKNOWLEDGEMENTS}

We thank Professors Morita, Kojima, Kawazumi, and Morifuji for their encouragement and advice.

\section{REFERENCES}

[Birman and Hilden 1973] J. S. Birman and H. M. Hilden, "On isotopies of homeomorphisms of Riemann surfaces", Ann. of Math. (2) 97 (1973), 424-439.

[Endo 2000] H. Endo, "Meyer's signature cocycle and hyperelliptic fibrations", Math. Ann. 316:2 (2000), 237-257.

[Humphries 1979] S. P. Humphries, "Generators for the mapping class group", pp. 44-47 in Topology of low-dimensional manifolds (Chelwood Gate, Sussex, 1977), edited by R. A. Fenn, Lecture Notes in Math. 722, Springer, Berlin, 1979.

[Jones 1987] V. F. R. Jones, "Hecke algebra representations of braid groups and link polynomials", Ann. of Math. (2) 126:2 (1987), 335-388.

[Kazhdan and Lusztig 1979] D. Kazhdan and G. Lusztig, "Representations of Coxeter groups and Hecke algebras", Invent. Math. 53:2 (1979), 165-184.

[Lickorish 1964] W. B. R. Lickorish, "A finite set of generators for the homeotopy group of a 2-manifold", Proc. Cambridge Philos. Soc. 60 (1964), 769-778. Corrigendum in 62 (1966), 679-681.

[Matsumoto 2000] M. Matsumoto, "A presentation of mapping class groups in terms of Artin groups and geometric monodromy of singularities", Math. Ann. 316:3 (2000), 401-418.

[Meyer 1973] W. Meyer, "Die Signatur von Flächenbündeln", Math. Ann. 201 (1973), 239-264.

[Ochiai and Kako 1995] M. Ochiai and F. Kako, "Computational construction of $W$-graphs of Hecke algebras $H(q, n)$ for $n$ up to 15", Experiment. Math. 4:1 (1995), 61-67.

[Penner and Harer 1992] R. C. Penner and J. L. Harer, Combinatorics of train tracks, Ann. Math. Studies 125, Princeton University Press, Princeton, NJ, 1992.

[Thurston 1988] W. P. Thurston, "On the geometry and dynamics of diffeomorphisms of surfaces", Bull. Amer. Math. Soc. (N.S.) 19:2 (1988), 417-431.

[Wajnryb 1983] B. Wajnryb, "A simple presentation for the mapping class group of an orientable surface", Israel J. Math. 45:2-3 (1983), 157-174.

[Wenzl 1988] H. Wenzl, "Hecke algebras of type $A_{n}$ and subfactors", Invent. Math. 92:2 (1988), 349-383. 


\begin{tabular}{|c|c|c|c|}
\hline$\zeta$ & $\varphi_{3}(\zeta)$ & $I(\zeta)$ & $J(\zeta)(q)$ \\
\hline$\{1\}$ & $2 / 7$ & $(-1+y)^{6}$ & $-\left(t^{9}-y\right)^{5}\left(1+t^{5} y\right)^{9} t^{-45}$ \\
\hline$\{1,1\}$ & $1 / 7$ & $(-1+y)^{6}$ & $-\left(t^{18}-y\right)^{5}\left(-1+t^{10} y\right)^{9} t^{-90}$ \\
\hline$\{1,2\}$ & $8 / 7$ & $(-1+y)^{4}\left(1-y+y^{2}\right)$ & $\left(-1+t^{10} y\right)^{4}\left(t^{8}+t^{4} y+y^{2}\right)^{5} t^{-40}$ \\
\hline$\{1,-2\}$ & 0 & $(-1+y)^{4}\left(1-y+y^{2}\right)$ & $-(1+y)^{4}\left(-t^{14}+y-t^{14} y+t^{28} y-t^{14} y^{2}\right)^{5} t^{-70}$ \\
\hline$\{1,3\}$ & $8 / 7$ & $(-1+y)^{6}$ & $\left(t^{18}-y\right)^{2}\left(t^{4}+y\right)^{6}\left(-1+t^{10} y\right)^{6} t^{-60}$ \\
\hline$\{1,-3\}$ & 0 & $(-1+y)^{6}$ & $\left(t^{14}-y\right)^{3}(1+y)^{8}\left(-1+t^{14} y\right)^{3} t^{-42}$ \\
\hline$\{1,1,1\}$ & $-2 / 7$ & $(-1+y)^{6}$ & $-\left(t^{27}-y\right)^{5}\left(1+t^{15} y\right)^{9} t^{-135}$ \\
\hline$\{1,1,2\}$ & $5 / 7$ & $(-1+y)^{4}\left(1+y^{2}\right)$ & $-\left(t^{6}-y\right)^{5}\left(t^{6}+y\right)^{5}\left(1+t^{15} y\right)^{4} t^{-60}$ \\
\hline$\{1,1,-2\}$ & $-3 / 7$ & $(-1+y)^{4}\left(1-4 y+y^{2}\right)$ & $-\left(-1+t^{5} y\right)^{4}\left(t^{23}-y+t^{14} y-t^{28} y+t^{42} y-t^{19} y^{2}\right)^{5} t^{-115}$ \\
\hline$\{1,1,3\}$ & $5 / 7$ & $(-1+y)^{6}$ & $\left(t^{27}-y\right)^{2}\left(t^{13}+y\right)^{3}(-1+t y)^{3}\left(1+t^{15} y\right)^{6} t^{-93}$ \\
\hline$\{1,1,-3\}$ & $-3 / 7$ & $(-1+y)^{6}$ & $-\left(t^{23}-y\right)^{3}\left(t^{9}+y\right)^{2}\left(-1+t^{5} y\right)^{6}\left(1+t^{19} y\right)^{3} t^{-87}$ \\
\hline$\{1,2,3\}$ & $12 / 7$ & $(-1+y)^{4}\left(1+y^{2}\right)$ & $\left(t^{6}-y\right)^{2}\left(t^{6}+y\right)^{2}(-1+t y)^{3}\left(1+t^{15} y\right)\left(1+t^{2} y^{2}\right)^{3} t^{-24}$ \\
\hline$\{1,2,-3\}$ & $4 / 7$ & $(-1+y)^{6}$ & $\begin{array}{l}\left(t^{9}+y\right)^{2}\left(-1+t^{5} y\right)^{3} \\
\quad \times\left(t^{18}+t^{9} y-t^{23} y+t^{37} y+y^{2}-t^{14} y^{2}+t^{28} y^{2}+t^{19} y^{3}\right)^{3} t^{-72}\end{array}$ \\
\hline$\{1,2,4\}$ & $12 / 7$ & $(-1+y)^{4}\left(1-y+y^{2}\right)$ & $(-1+t y)\left(1+t^{15} y\right)^{3}\left(t^{26}+t^{13} y+y^{2}\right)^{2}\left(1-t y+t^{2} y^{2}\right)^{3} t^{-52}$ \\
\hline$\{1,2,-4\}$ & $4 / 7$ & $(-1+y)^{4}\left(1-y+y^{2}\right)$ & $\left(1+t^{5} y\right)^{3}\left(-1+t^{19} y\right)\left(t^{18}-t^{9} y+y^{2}\right)^{3}\left(1+t^{5} y+t^{10} y^{2}\right)^{2} t^{-54}$ \\
\hline$\{1,-2,3\}$ & $4 / 7$ & $(-1+y)^{4}\left(1-4 y+y^{2}\right)$ & $\begin{array}{l}\left(t^{9}+y\right)^{3}\left(-1+t^{5} y\right)\left(t^{23}-y+t^{14} y-t^{28} y+t^{42} y-t^{19} y^{2}\right)^{2} \\
\quad \times\left(t^{9}+y-2 t^{14} y+t^{28} y+t^{19} y^{2}\right)^{3} t^{-100}\end{array}$ \\
\hline$\{1,-2,4\}$ & $4 / 7$ & $(-1+y)^{4}\left(1-3 y+y^{2}\right)$ & $\begin{array}{l}\left(t^{9}+y\right)\left(-1+t^{5} y\right)^{3}\left(-t^{23}+y-t^{14} y+t^{28} y-t^{5} y^{2}\right)^{2} \\
\quad \times\left(t^{9}+y-t^{14} y+t^{28} y+t^{19} y^{2}\right)^{3} t^{-82}\end{array}$ \\
\hline$\{1,3,5\}$ & $12 / 7$ & $(-1+y)^{6}$ & $\left(t^{27}-y\right)\left(t^{13}+y\right)^{3}(-1+t y)^{6}\left(1+t^{15} y\right)^{4} t^{-66}$ \\
\hline$\{1,3,-5\}$ & $4 / 7$ & $(-1+y)^{6}$ & $-\left(t^{23}-y\right)\left(t^{9}+y\right)^{5}\left(-1+t^{5} y\right)^{6}\left(1+t^{19} y\right)^{2} t^{-68}$ \\
\hline$\{1,1,1,1\}$ & $-5 / 7$ & $(-1+y)^{6}$ & $-\left(t^{36}-y\right)^{5}\left(-1+t^{20} y\right)^{9} t^{-180}$ \\
\hline$\{1,1,1,2\}$ & $2 / 7$ & $(-1+y)^{4}\left(1+y+y^{2}\right)$ & $\left(-1+t^{20} y\right)^{4}\left(t^{16}+t^{8} y+y^{2}\right)^{5} t^{-80}$ \\
\hline$\{1,1,1,-2\}$ & $-6 / 7$ & $(-1+y)^{4}\left(1-5 y+y^{2}\right)$ & $-\left(1+t^{10} y\right)^{4}\left(-t^{32}+y-t^{14} y+t^{28} y-t^{42} y+t^{56} y-t^{24} y^{2}\right)^{5} t^{-160}$ \\
\hline$\{1,1,1,3\}$ & $2 / 7$ & $(-1+y)^{6}$ & $\left(t^{36}-y\right)^{2}\left(t^{22}+y\right)^{3}\left(1+t^{6} y\right)^{3}\left(-1+t^{20} y\right)^{6} t^{-138}$ \\
\hline$\{1,1,1,-3\}$ & $-6 / 7$ & $(-1+y)^{6}$ & $-\left(t^{32}-y\right)^{3}\left(t^{18}+y\right)^{2}\left(1+t^{10} y\right)^{6}\left(-1+t^{24} y\right)^{3} t^{-132}$ \\
\hline$\{1,1,2,2\}$ & $2 / 7$ & $(-1+y)^{4}(1+y)^{2}$ & $-\left(t^{22}-y\right)^{5}\left(-1+t^{6} y\right)^{5}\left(-1+t^{20} y\right)^{4} t^{-110}$ \\
\hline$\{1,1,2,3\}$ & $9 / 7$ & $(-1+y)^{4}\left(1+y+y^{2}\right)$ & $\left(-1+t^{20} y\right)\left(t^{16}+t^{8} y+y^{2}\right)^{2}\left(-1+t^{4} y^{3}\right)^{3} t^{-32}$ \\
\hline$\{1,1,2,-3\}$ & $1 / 7$ & $(-1+y)^{4}\left(1-y+y^{2}\right)$ & $-\left(1+t^{10} y\right)\left(t^{8}-t^{4} y+y^{2}\right)^{2}\left(-t^{22}-t^{32} y+t^{46} y+y^{2}-t^{14} y^{2}-t^{24} y^{3}\right)^{3} t^{-82}$ \\
\hline$\{1,1,2,4\}$ & $9 / 7$ & $(-1+y)^{4}\left(1+y^{2}\right)$ & $-(t-y)^{3}\left(t^{15}-y\right)^{2}(t+y)^{3}\left(t^{15}+y\right)^{2}\left(1+t^{6} y\right)\left(-1+t^{20} y\right)^{3} t^{-66}$ \\
\hline$\{1,1,2,-4\}$ & $1 / 7$ & $(-1+y)^{4}\left(1+y^{2}\right)$ & $-\left(t^{11}-y\right)^{3}\left(t^{11}+y\right)^{3}\left(-1+t^{3} y\right)^{2}\left(1+t^{3} y\right)^{2}\left(-1+t^{10} y\right)^{3}\left(1+t^{24} y\right) t^{-66}$ \\
\hline$\{1,1,-2,-2\}$ & 0 & $(-1+y)^{4}\left(1-6 y+y^{2}\right)$ & $-(-1+y)^{4}\left(-t^{28}+y-t^{14} y+2 t^{28} y-t^{42} y+t^{56} y-t^{28} y^{2}\right)^{5} t^{-140}$ \\
\hline$\{1,1,-2,3\}$ & $1 / 7$ & $(-1+y)^{4}\left(1-5 y+y^{2}\right)$ & $\begin{array}{l}-\left(1+t^{10} y\right)\left(-t^{32}+y-t^{14} y+t^{28} y-t^{42} y+t^{56} y-t^{24} y^{2}\right)^{2} \\
\quad \times\left(-t^{22}-t^{4} y+2 t^{18} y-2 t^{32} y+t^{46} y+y^{2}-2 t^{14} y^{2}+2 t^{28} y^{2}\right. \\
\left.\quad-t^{42} y^{2}-t^{24} y^{3}\right)^{3} t^{-130}\end{array}$ \\
\hline
\end{tabular}

TABLE 3. Data for the case $g=3$ : Meyer's function $\varphi_{3}$ and the polynomials $I(\zeta)=\operatorname{det}\left(y E_{6}-\operatorname{Sp}(\zeta)\right)$ and $J(\zeta)=\operatorname{det}\left(y E_{14}-\rho_{3}(\zeta)\right)$. We use $t:=q^{1 / 14}$ for simplicity. The Thurston type is reducible in every case. 


\begin{tabular}{|c|c|c|c|}
\hline$\zeta$ & $\varphi_{3}(\zeta)$ & $I(\zeta)$ & $J(\zeta)(q)$ \\
\hline$\{1,1,-2,-3\}$ & -1 & $(-1+y)^{4}\left(1-3 y+y^{2}\right)$ & $\begin{array}{l}-(-1+y) \\
\quad \times\left(t^{14}+y-t^{14} y+t^{28} y+t^{14} y^{2}\right)^{2}-\left(t^{28}-y+t^{14} y-t^{28} y+t^{42} y-t^{14} y^{2}\right. \\
\left.\quad+t^{28} y^{2}-t^{42} y^{2}+t^{56} y^{2}-t^{28} y^{3}\right)^{3} t^{-112}\end{array}$ \\
\hline$\{1,1,-2,4\}$ & $1 / 7$ & $(-1+y)^{4}\left(1-4 y+y^{2}\right)$ & $\begin{array}{l}-\left(t^{4}-y\right)\left(1+t^{10} y\right)^{3}\left(t^{32}-y+t^{14} y-t^{28} y+t^{42} y-t^{10} y^{2}\right)^{2} \\
\quad \times\left(-t^{18}-y+t^{14} y-t^{28} y+t^{42} y+t^{24} y^{2}\right)^{3} t^{-122}\end{array}$ \\
\hline$\{1,1,-2,-4\}$ & -1 & $(-1+y)^{4}\left(1-4 y+y^{2}\right)$ & $\begin{array}{l}(1+y)^{3}\left(-1+t^{14} y\right)\left(-t^{28}-y+t^{14} y-t^{28} y+t^{42} y+t^{14} y^{2}\right)^{3} \\
\quad \times\left(t^{14}-y+t^{14} y-t^{28} y+t^{42} y-t^{28} y^{2}\right)^{2} t^{-112}\end{array}$ \\
\hline$\{1,1,3,3\}$ & $2 / 7$ & $(-1+y)^{6}$ & $\left(t^{8}-y\right)^{6}\left(t^{36}-y\right)^{2}\left(-1+t^{20} y\right)^{6} t^{-120}$ \\
\hline$\{1,1,3,4\}$ & $9 / 7$ & $(-1+y)^{4}\left(1-y+y^{2}\right)$ & $-\left(t^{8}-y\right)\left(-1+t^{20} y\right)^{3}\left(t^{44}+t^{22} y+y^{2}\right)^{2}\left(1+t^{6} y+t^{12} y^{2}\right)^{3} t^{-96}$ \\
\hline$\{1,1,3,-4\}$ & $1 / 7$ & $(-1+y)^{4}\left(1-3 y+y^{2}\right)$ & $\begin{array}{l}-\left(t^{18}-y\right)\left(-1+t^{10} y\right)^{3}\left(t^{36}+t^{4} y-t^{18} y+t^{32} y+y^{2}\right)^{2} \\
\quad \times\left(t^{4}+y-t^{14} y+t^{28} y+t^{24} y^{2}\right)^{3} t^{-102}\end{array}$ \\
\hline$\{1,1,3,5\}$ & $9 / 7$ & $(-1+y)^{6}$ & $\left(t^{8}-y\right)^{3}\left(t^{36}-y\right)\left(t^{22}+y\right)^{2}\left(1+t^{6} y\right)^{4}\left(-1+t^{20} y\right)^{4} t^{-104}$ \\
\hline$\{1,1,3,-5\}$ & $1 / 7$ & $(-1+y)^{6}$ & $\left(t^{4}-y\right)^{3}\left(t^{32}-y\right)\left(t^{18}+y\right)^{3}\left(1+t^{10} y\right)^{5}\left(-1+t^{24} y\right)^{2} t^{-98}$ \\
\hline$\{1,1,-3,-3\}$ & 0 & $(-1+y)^{6}$ & $-\left(t^{28}-y\right)^{3}(-1+y)^{8}\left(-1+t^{28} y\right)^{3} t^{-84}$ \\
\hline$\{1,1,-3,-4\}$ & -1 & $(-1+y)^{4}\left(1-y+y^{2}\right)$ & $(1+y)^{3}\left(t^{28}+y\right)\left(t^{28}-t^{14} y+y^{2}\right)^{2}\left(1-t^{14} y+t^{28} y^{2}\right)^{3} t^{-84}$ \\
\hline$\{1,1,-3,-5\}$ & -1 & $(-1+y)^{6}$ & $\left(t^{28}-y\right)^{2}(-1+y)^{5}\left(t^{14}+y\right)^{2}\left(1+t^{14} y\right)^{4}\left(-1+t^{28} y\right) t^{-84}$ \\
\hline$\{1,2,2,3\}$ & $9 / 7$ & $(-1+y)^{4}(1+y)^{2}$ & $\left(t^{22}-y\right)^{2}\left(t^{8}+y\right)^{3}\left(-1+t^{6} y\right)^{5}\left(1+t^{6} y\right)^{3}\left(-1+t^{20} y\right) t^{-68}$ \\
\hline$\{1,2,2,-3\}$ & $1 / 7$ & $(-1+y)^{6}$ & $\begin{array}{l}\left(t^{18}+y\right)^{2}\left(1+t^{10} y\right)^{3} \\
\quad \times\left(-t^{22}+t^{18} y-t^{32} y+t^{46} y+y^{2}-t^{14} y^{2}+t^{28} y^{2}-t^{24} y^{3}\right)^{3} t^{-102}\end{array}$ \\
\hline$\{1,2,3,4\}$ & $16 / 7$ & $\begin{array}{r}(-1+y)^{2} \\
\times\left(1-y+y^{2}-y^{3}+y^{4}\right)\end{array}$ & $\left(1+t^{6} y+t^{12} y^{2}+t^{18} y^{3}+t^{24} y^{4}\right)\left(t^{12}-y^{5}\right)^{2} t^{-24}$ \\
\hline$\{1,2,3,-4\}$ & $8 / 7$ & $\begin{array}{r}(-1+y)^{2} \\
\times\left(1-3 y+3 y^{2}-3 y^{3}+y^{4}\right)\end{array}$ & $\begin{array}{l}\left(t^{12}+t^{8} y-t^{22} y+t^{36} y+t^{4} y^{2}-t^{18} y^{2}+t^{32} y^{2}+y^{3}-t^{14} y^{3}+t^{28} y^{3}+t^{24} y^{4}\right) \\
\times\left(t^{26}+t^{22} y-t^{36} y-t^{4} y^{2}+2 t^{18} y^{2}-2 t^{32} y^{2}+t^{46} y^{2}-y^{3}+2 t^{14} y^{3}\right. \\
\left.\quad-2 t^{28} y^{3}+t^{42} y^{3}+t^{10} y^{4}-t^{24} y^{4}-t^{20} y^{5}\right)^{2} t^{-64}\end{array}$ \\
\hline$\{1,2,3,5\}$ & $16 / 7$ & $(-1+y)^{4}\left(1+y^{2}\right)$ & $\begin{array}{l}-(t-y)\left(t^{8}-y\right)\left(t^{15}-y\right)(t+y)\left(t^{15}+y\right)\left(1+t^{6} y\right)^{2}\left(-1+t^{20} y\right)\left(t^{16}+y^{2}\right) \\
\quad \times\left(1+t^{12} y^{2}\right)^{2} t^{-56}\end{array}$ \\
\hline$\{1,2,3,-5\}$ & $8 / 7$ & $(-1+y)^{4}\left(1+y^{2}\right)$ & $\begin{array}{l}-\left(t^{4}-y\right)^{2}\left(t^{11}-y\right)\left(t^{11}+y\right)\left(-1+t^{3} y\right)\left(1+t^{3} y\right)\left(1+t^{10} y\right)^{2}\left(t^{8}+y^{2}\right)^{2} \\
\quad \times\left(1+t^{20} y^{2}\right) t^{-46}\end{array}$ \\
\hline$\{1,2,-3,4\}$ & $8 / 7$ & $\begin{array}{r}(-1+y)^{2} \\
\times\left(1-5 y+7 y^{2}-5 y^{3}+y^{4}\right)\end{array}$ & $\begin{array}{l}-\left(-t^{12}+2 t^{8} y-2 t^{22} y+t^{36} y-2 t^{4} y^{2}+3 t^{18} y^{2}-2 t^{32} y^{2}+y^{3}-2 t^{14} y^{3}\right. \\
\left.\quad+2 t^{28} y^{3}-t^{24} y^{4}\right) \\
\times\left(-t^{40}-t^{22} y+2 t^{36} y-2 t^{50} y+t^{64} y-t^{4} y^{2}+3 t^{18} y^{2}-5 t^{32} y^{2}+5 t^{46} y^{2}\right. \\
\quad-3 t^{60} y^{2}+t^{74} y^{2}+y^{3}-3 t^{14} y^{3}+5 t^{28} y^{3}-5 t^{42} y^{3}+3 t^{56} y^{3}-t^{70} y^{3} \\
\left.\quad+t^{10} y^{4}-2 t^{24} y^{4}+2 t^{38} y^{4}-t^{52} y^{4}-t^{34} y^{5}\right)^{2} t^{-92}\end{array}$ \\
\hline$\{1,2,-3,-4\}$ & 0 & $\begin{array}{r}(-1+y)^{2} \\
\times\left(1-3 y+5 y^{2}-3 y^{3}+y^{4}\right)\end{array}$ & $\begin{array}{l}(1+y)^{2}\left(-t^{14}+y-t^{14} y+t^{28} y-t^{14} y^{2}+y^{3}-t^{14} y^{3}+t^{28} y^{3}-t^{14} y^{4}\right)^{2} \\
\quad \times\left(t^{28}-t^{14} y+t^{28} y-t^{42} y+y^{2}-t^{14} y^{2}+t^{28} y^{2}-t^{42} y^{2}+t^{56} y^{2}\right. \\
\left.\quad-t^{14} y^{3}+t^{28} y^{3}-t^{42} y^{3}+t^{28} y^{4}\right) t^{-56}\end{array}$ \\
\hline$\{1,2,-3,5\}$ & $8 / 7$ & $(-1+y)^{6}$ & $\begin{array}{l}\left(t^{4}-y\right)^{2}\left(t^{18}+y\right)\left(1+t^{10} y\right)^{2} \\
\quad \times\left(t^{36}+t^{18} y-t^{32} y+t^{46} y+y^{2}-t^{14} y^{2}+t^{28} y^{2}+t^{10} y^{3}\right) \\
\quad \times\left(-t^{8}+t^{4} y-t^{18} y+t^{32} y-y^{2}+t^{14} y^{2}-t^{28} y^{2}+t^{24} y^{3}\right)^{2} t^{-78}\end{array}$ \\
\hline$\{1,2,-3,-5\}$ & 0 & $(-1+y)^{6}$ & $\begin{array}{l}(-1+y)^{3}\left(t^{14}+y\right)\left(1+t^{14} y\right) \\
\quad \times\left(t^{28}+t^{14} y-t^{28} y+t^{42} y+y^{2}-t^{14} y^{2}+t^{28} y^{2}+t^{14} y^{3}\right)^{2} \\
\quad \times\left(-1+y-t^{14} y+t^{28} y-y^{2}+t^{14} y^{2}-t^{28} y^{2}+t^{28} y^{3}\right) t^{-70}\end{array}$ \\
\hline
\end{tabular}

TABLE 3 (continued) 


\begin{tabular}{|c|c|c|c|}
\hline$\zeta$ & $\varphi_{3}(\zeta)$ & $I(\zeta)$ & $J(\zeta)(q)$ \\
\hline$\{1,2,4,5\}$ & $16 / 7$ & $(-1+y)^{2}\left(1-y+y^{2}\right)^{2}$ & $\left(t^{8}-y\right)^{4}\left(-1+t^{20} y\right)^{2}\left(t^{16}+t^{8} y+y^{2}\right)^{2}\left(1+t^{6} y+t^{12} y^{2}\right)^{2} t^{-64}$ \\
\hline$\{1,2,4,-5\}$ & $8 / 7$ & $\times\left(1-3 y+y^{2}\right)\left(1-y+y^{2}\right)$ & $\begin{array}{l}-\left(1+t^{10} y\right)^{2}\left(t^{8}-t^{4} y+y^{2}\right)\left(-t^{4}+y-t^{14} y+t^{28} y-t^{24} y^{2}\right) \\
\quad \times\left(t^{36}+t^{18} y-t^{32} y+t^{46} y+y^{2}-2 t^{14} y^{2}+2 t^{28} y^{2}-2 t^{42} y^{2}+t^{56} y^{2}\right. \\
\left.\quad+t^{10} y^{3}-t^{24} y^{3}+t^{38} y^{3}+t^{20} y^{4}\right)^{2} t^{-84}\end{array}$ \\
\hline$\{1,2,4,6\}$ & $16 / 7$ & $(-1+y)^{4}\left(1-y+y^{2}\right)$ & $\begin{array}{l}\left(1+t^{6} y\right)^{2}\left(-1+t^{20} y\right)^{2}\left(t^{16}-t^{8} y+y^{2}\right)^{2}\left(t^{44}+t^{22} y+y^{2}\right) \\
\quad \times\left(1+t^{6} y+t^{12} y^{2}\right)^{2} t^{-76}\end{array}$ \\
\hline$\{1,2,4,-6\}$ & $8 / 7$ & $(-1+y)^{4}\left(1-y+y^{2}\right)$ & $\begin{array}{l}-\left(t^{4}-y\right)\left(1+t^{10} y\right)^{2}\left(-1+t^{24} y\right)\left(t^{8}-t^{4} y+y^{2}\right)^{3}\left(t^{36}+t^{18} y+y^{2}\right) \\
\quad \times\left(1+t^{10} y+t^{20} y^{2}\right) t^{-64}\end{array}$ \\
\hline$\{1,2,-4,-5\}$ & 0 & $(-1+y)^{2}\left(1-y+y^{2}\right)^{2}$ & $(1+y)^{6}\left(1-y+y^{2}\right)^{2}\left(t^{28}-t^{14} y+y^{2}\right)\left(1-t^{14} y+t^{28} y^{2}\right) t^{-28}$ \\
\hline$\{1,2,-4,-6\}$ & 0 & $(-1+y)^{4}\left(1-y+y^{2}\right)$ & $\begin{array}{l}(1+y)^{2}\left(-1+t^{14} y\right)^{2}\left(1+y+y^{2}\right)^{2}\left(t^{28}-t^{14} y+y^{2}\right)^{2} \\
\quad \times\left(1-t^{14} y+t^{28} y^{2}\right) t^{-56}\end{array}$ \\
\hline$\{1,-2,1,-2\}$ & 0 & $(-1+y)^{4}\left(1-7 y+y^{2}\right)$ & $-(-1+y)^{4}\left(-t^{28}+y-2 t^{14} y+t^{28} y-2 t^{42} y+t^{56} y-t^{28} y^{2}\right)^{5} t^{-140}$ \\
\hline$\{1,-2,-2,3\}$ & 1 & $(-1+y)^{4}\left(1-6 y+y^{2}\right)$ & $\begin{array}{l}(-1+y)\left(t^{14}+y\right)^{3}\left(-t^{28}+y-t^{14} y+2 t^{28} y-t^{42} y+t^{56} y-t^{28} y^{2}\right)^{2} \\
\quad \times\left(-t^{14}-y+2 t^{14} y-2 t^{28} y+t^{42} y+t^{28} y^{2}\right)^{3} t^{-140}\end{array}$ \\
\hline$\{1,-2,3,-2\}$ & 1 & $(-1+y)^{4}\left(1-7 y+y^{2}\right)$ & $\begin{array}{l}(-1+y)\left(-t^{28}+y-2 t^{14} y+t^{28} y-2 t^{42} y+t^{56} y-t^{28} y^{2}\right)^{2} \\
\quad \times\left(-t^{28}-2 t^{14} y+3 t^{28} y-2 t^{42} y+t^{56} y-y^{2}+2 t^{14} y^{2}-3 t^{28} y^{2}\right. \\
\left.\quad+2 t^{42} y^{2}+t^{28} y^{3}\right)^{3} t^{-140}\end{array}$ \\
\hline$\{1,-2,3,-4\}$ & 0 & $\begin{array}{r}(-1+y)^{2} \\
\times\left(1-7 y+13 y^{2}-7 y^{3}+y^{4}\right)\end{array}$ & $\begin{aligned}(1+y)^{2} & \left(t^{28}-2 t^{14} y+3 t^{28} y-2 t^{42} y+y^{2}-3 t^{14} y^{2}+5 t^{28} y^{2}-3 t^{42} y^{2}\right. \\
& \left.+t^{56} y^{2}-2 t^{14} y^{3}+3 t^{28} y^{3}-2 t^{42} y^{3}+t^{28} y^{4}\right) \\
\times & \left(-t^{42}-t^{14} y+3 t^{28} y-3 t^{42} y+3 t^{56} y-t^{70} y+y^{2}-3 t^{14} y^{2}\right. \\
& +5 t^{28} y^{2}-7 t^{42} y^{2}+5 t^{56} y^{2}-3 t^{70} y^{2}+t^{84} y^{2}-t^{14} y^{3} \\
& \left.+3 t^{28} y^{3}-3 t^{42} y^{3}+3 t^{56} y^{3}-t^{70} y^{3}-t^{42} y^{4}\right)^{2} t^{-112}\end{aligned}$ \\
\hline$\{1,-2,3,5\}$ & $8 / 7$ & $(-1+y)^{4}\left(1-4 y+y^{2}\right)$ & $\begin{array}{l}-\left(t^{4}-y\right)^{2}\left(t^{18}+y\right)\left(1+t^{10} y\right)\left(t^{32}-y+t^{14} y-t^{28} y+t^{42} y-t^{10} y^{2}\right) \\
\quad \times\left(t^{18}+y-2 t^{14} y+t^{28} y+t^{10} y^{2}\right)\left(-t^{4}+y-2 t^{14} y+t^{28} y-t^{24} y^{2}\right)^{2} \\
\quad \times\left(-t^{18}-y+t^{14} y-t^{28} y+t^{42} y+t^{24} y^{2}\right) t^{-102}\end{array}$ \\
\hline$\{1,-2,3,-5\}$ & 0 & $(-1+y)^{4}\left(1-4 y+y^{2}\right)$ & $\begin{array}{l}-(-1+y)^{2}\left(t^{14}+y\right)^{2}\left(t^{28}-y+t^{14} y-t^{28} y+t^{42} y-t^{14} y^{2}\right) \\
\quad \times\left(t^{14}+y-2 t^{14} y+t^{28} y+t^{14} y^{2}\right)^{2}\left(1-y+2 t^{14} y-t^{28} y+t^{28} y^{2}\right) \\
\quad \times\left(-t^{14}-y+t^{14} y-t^{28} y+t^{42} y+t^{28} y^{2}\right) t^{-98}\end{array}$ \\
\hline$\{1,-2,-3,4\}$ & 0 & $\begin{array}{r}(-1+y)^{2} \\
\times\left(1-5 y+9 y^{2}-5 y^{3}+y^{4}\right)\end{array}$ & $\begin{array}{l}(-1+y)^{2}\left(-t^{28}+y-2 t^{14} y+2 t^{28} y-2 t^{42} y-y^{2}+2 t^{14} y^{2}-3 t^{28} y^{2}\right. \\
\left.\quad+2 t^{42} y^{2}-t^{56} y^{2}-2 t^{14} y^{3}+2 t^{28} y^{3}-2 t^{42} y^{3}+t^{56} y^{3}-t^{28} y^{4}\right)^{2} \\
\times\left(t^{28}+2 t^{14} y-2 t^{28} y+t^{42} y+y^{2}-2 t^{14} y^{2}+3 t^{28} y^{2}-2 t^{42} y^{2}\right. \\
\left.\quad+t^{56} y^{2}+t^{14} y^{3}-2 t^{28} y^{3}+2 t^{42} y^{3}+t^{28} y^{4}\right) t^{-84}\end{array}$ \\
\hline$\{1,-2,4,-5\}$ & 0 & $(-1+y)^{2}\left(1-3 y+y^{2}\right)^{2}$ & $\begin{array}{l}(-1+y)^{6}\left(t^{14}+y-t^{14} y+t^{28} y+t^{14} y^{2}\right)^{2} \\
\quad \times\left(-t^{28}+y-2 t^{14} y+t^{28} y-2 t^{42} y+t^{56} y-t^{28} y^{2}\right)^{2} t^{-84}\end{array}$ \\
\hline$\{1,-2,4,6\}$ & $8 / 7$ & $(-1+y)^{4}\left(1-3 y+y^{2}\right)$ & $\begin{array}{l}\left(t^{4}-y\right)^{2}\left(1+t^{10} y\right)^{2}\left(t^{36}-t^{4} y+t^{18} y-t^{32} y+y^{2}\right) \\
\quad \times\left(t^{18}+y-t^{14} y+t^{28} y+t^{10} y^{2}\right)^{2}\left(-t^{4}+y-t^{14} y+t^{28} y-t^{24} y^{2}\right)^{2} \\
\quad \times t^{-88}\end{array}$ \\
\hline$\{1,-2,4,-6\}$ & 0 & $(-1+y)^{4}\left(1-3 y+y^{2}\right)$ & $\begin{array}{l}-(-1+y)^{2}\left(t^{14}+y\right)\left(1+t^{14} y\right)\left(-t^{28}+y-t^{14} y+t^{28} y-y^{2}\right) \\
\quad \times\left(t^{14}+y-t^{14} y+t^{28} y+t^{14} y^{2}\right)^{3}\left(1-y+t^{14} y-t^{28} y+t^{28} y^{2}\right) t^{-84}\end{array}$ \\
\hline$\{1,3,5,7\}$ & $9 / 7$ & $(-1+y)^{6}$ & $\left(t^{8}-y\right)^{6}\left(t^{36}-y\right)\left(1+t^{6} y\right)^{4}\left(-1+t^{20} y\right)^{3} t^{-84}$ \\
\hline$\{1,3,5,-7\}$ & $1 / 7$ & $(-1+y)^{6}$ & $-\left(t^{4}-y\right)^{3}\left(t^{18}+y\right)^{4}\left(1+t^{10} y\right)^{6}\left(-1+t^{24} y\right) t^{-84}$ \\
\hline$\{1,3,-5,-7\}$ & 0 & $(-1+y)^{6}$ & $-\left(t^{28}-y\right)(-1+y)^{8}\left(t^{14}+y\right)^{2}\left(1+t^{14} y\right)^{2}\left(-1+t^{28} y\right) t^{-56}$ \\
\hline
\end{tabular}

TABLE 3 (continued) 
Kazushi Ahara, Department of Mathematics, Meiji University, Higashimita 1-1-1, Tama, Kawasaki, Kanagawa, Japan 214-8571 (ahara@math.meiji.ac.jp)

Mitsuhiko Takasawa, Department of Mathematical and Computing Sciences, Tokyo Institute of Technology, 2-12-1 Ookayama, Meguro-ku, Tokyo, Japan 152-8551 (takasawa@is.titech.ac.jp)

Received August 3, 1999; accepted November 15, 1999 